\title{
The Implied Volatility Smirk
}

\author{
Jin E. Zhang ${ }^{1}$ \\ School of Economics and Finance \\ and School of Business \\ The University of Hong Kong \\ Pokfulam Road, Hong Kong \\ Email: jinzhang@hku.hk \\ Yi Xiang \\ Department of Finance \\ Hong Kong University of Science and Technology \\ Clear Water Bay, Kowloon, Hong Kong \\ Email: hkxy@ust.hk
}

First Version: December 2003

Final Version: December 2006

\section{Forthcoming in Quantitative Finance}

This paper provides an industry standard on how to quantify the shape of the implied volatility smirk in the equity index options market. Our local expansion method uses a second-order polynomial to describe the implied volatility - moneyness function and relates the coefficients of the polynomial to the properties of the implied risk-neutral distribution of the equity index return. We present a formal, two-way representation of the link between the level, slope and curvature of the implied volatility smirk and the risk-neutral standard deviation, skewness and excess kurtosis. We then propose a new semi-analytical method to calibrate option-pricing models based on the quantified implied volatility smirk, and investigate the applicability of two option-pricing models.

Keywords: Option pricing; Implied volatility smirk; Risk-neutral skewness and excess kurtosis; Term structure

JEL Classification Code: G13

\footnotetext{
${ }^{1}$ Corresponding author. Tel: (852) 2859 1033, Fax: (852) 2548 1152. We are especially grateful to the two referees Mark Schroder and Liuren Wu whose helpful comments substantially improved the paper. We also acknowledge helpful comments from Jean-Philippe Bouchaud (co-editor-in-chief), Charles Cao, Peter Carr, Andrew P. Carverhill, Eric C. Chang, Bruno Dupire, Patrick S. Hagan, Jing-zhi Huang (our CICF discussant), Jun Pan, James J.-D. Wang, Chu Zhang, and seminar participants at the University of Hong Kong (HKU), Hong Kong University of Science and Technology (HKUST), Bloomberg, L. P., Claremont Graduate University, Shanghai University of Finance and Economics, Tongji University, University of Science and Technology of China, and 2005 China International Conference in Finance (CICF 2005) in Kunming. The research for this paper was partially supported by HKUST under the Direct Allocation Grant (Project No. DAG03/04.BM53), by HKU under the Small Project Funding scheme (Project No. 200507176196), and by the Research Grants Council of Hong Kong under the CERG grant (Project No. HKU $7427 / 06 \mathrm{H})$.
} 


\title{
The Implied Volatility Smirk
}

\begin{abstract}
This paper provides an industry standard on how to quantify the shape of the implied volatility smirk in the equity index options market. Our local expansion method uses a second-order polynomial to describe the implied volatility - moneyness function and relates the coefficients of the polynomial to the properties of the implied risk-neutral distribution of the equity index return. We present a formal, two-way representation of the link between the level, slope and curvature of the implied volatility smirk and the risk-neutral standard deviation, skewness and excess kurtosis. We then propose a new semi-analytical method to calibrate option-pricing models based on the quantified implied volatility smirk, and investigate the applicability of two option-pricing models.
\end{abstract}




\section{Introduction}

The implied volatility of an option is defined as the volatility that equates the Black-Scholes (1973) formula to the market price of the option. It is another way to quote the option price. The implied volatility on a particular day, as a two-dimensional function of the strike price and time to maturity, is regarded as the implied volatility surface. Rubinstein (1985) documented the phenomenon of the implied volatility smile before 1987, i.e., the implied volatility of US equity index options, as a function of the strike price for a certain maturity, followed the pattern of a symmetric and smiling curve. Since the market crash in 1987, the implied volatility as a function of the strike price has been skewed towards the left, see, e.g., the empirical evidence of Rubinstein (1994), Aït-Sahalia and Lo (1998), and Foresi and $\mathrm{Wu}(2005)$. This phenomenon has been called the implied volatility smirk. ${ }^{2}$

Market smirks are usually managed by using the local volatility model of Derman and Kani (1994), Dupire (1994) and Rubinstein (1994) in the financial industry. Ncube (1996) fits the implied volatility surface of FTSE 100 index options with parameter models. Dumas, Fleming and Whaley (1998) examines the predictive and hedging performance of option-pricing models under deterministic (local) volatility by using S\&P 500 options from June 1988 through December 1993. They find it is no better than an ad hoc procedure that merely smooths the implied volatility across strike prices and times to maturity. Skiadopoulos, Hodges and Clewlow (1999) analyzes the dynamics of the implied volatility smile of S\&P 500 futures options by applying principal components analysis. Peña, Rubio and Serna (1999) studies the determinants of the implied volatility function, such as transaction costs, time to expiration, market uncertainty and relative market momentum. They use the Spanish IBEX-35 index options from January 1994 to April 1996. Hagan et al. (2002) finds that the dynamics of the market smile (or smirk) predicted by the local volatility model is opposite of the observed market behavior. They propose a stochastic volatility constant-

\footnotetext{
${ }^{2}$ Both terms, implied volatility smirk and skew are used in the finance literature and the financial industry with slightly different meaning. A smirk can be regarded as the superposition of a skew and a smile.
} 
elasticity-of-variance $(\mathrm{CEV})$ model $^{3}$ for the forward price and price European options by using singular perturbation method. Cont and da Fonseca (2002) studies the evolution of entire implied volatility surface. Dennis and Mayhew (2002) empirically studies the cross-sectional behavior of the implied volatility smirk, in particular the relation between the risk-neutral skewness implied by individual stock options and the beta of the stock. Jiang et al. (2003) recovers the implied local volatility numerically in an optimal control framework. Bollen and Whaley (2004) studies the source of the implied volatility smirk, in particular the effect of net buying pressure. Foresi and Wu (2005) documented the phenomenon of the implied volatility smirk for the major equity indices in twelve countries. Brenner, Ou and Zhang (2006) proposes using derivatives on the at-the-money-forward straddle to trade and hedge volatility risk.

The implied volatility surface has all the information of market option prices. It reflects the risk-neutral distribution of underlying asset returns over different horizons. Understanding and making good use of it are very important. These are the subjects of this paper. Our target is to develop a simple and intuitive way to quantify the shape of the implied volatility smirk and an easy-to-use method to calibrate option-pricing models.

Since most of the traded options are near the money, we will look closely at the implied volatility smirk in the neighborhood of the strike at the money ${ }^{4}$. We define the moneyness to be the logarithm of the strike price over the implied forward price, normalized by the standard deviation of the return on maturity. We use a second-order polynomial function to describe the implied volatility - moneyness curve, and we relate the level, the slope, and the curvature coefficients to the properties of the implied risk-neutral distribution of the asset return. The main contribution of this paper lies in a formal, two-way representation of the link between the level, slope and curvature of the implied volatility smirk and the risk-neutral standard deviation, skewness and excess kurtosis.

We document the term structure and dynamics of the level, slope and curvature of

\footnotetext{
${ }^{3}$ The model is known as the SABR model, which stands for stochastic alpha beta rho (Hagan et al. 2002).

${ }^{4}$ In this paper, at the money is defined as the point that the strike price, $K$, is equal to the implied forward price, $F_{0}$.
} 
the implied volatility smirk of the S\&P 500 index options market. On a randomly-picked date, November 4, 2003, the term structure of the level, i.e., the at-the-money implied volatility (ATMV), of November S\&P 500 index options was upward sloping. The value of the implied volatility ranged from $14 \%$ for two-week short-term options to $18 \%$ for longterm options with an observable horizon of two years. The term structure of the slope is almost flat especially for long-term options. The value of the slope is about -0.17 , very stable for options with maturities ranging from two months to two years. The negative value of the slope implies the negative skewness of the risk-neutral distribution of S\&P 500 index returns. The implied volatility smirk does not flatten out as the maturity increases to almost two years. This pattern has been documented and explained with a Finite Moment Log Stable (FMLS) process by Carr and Wu (2003). The term structure of the curvature oscillates between 0 and 0.04 . We also observe that the term structure of the ATMV does not change much on a daily basis. It has a more regular pattern than the slope and the curvature. This indicates that investors have a better understanding about the ATMV than about the slope and the curvature. Our empirical results also show that the time series of the level, slope and curvature of the implied volatility smirk for options with a fixed maturity date is quite stationary.

We then use the term structure information to calibrate two option-pricing models: the Constant Elasticity Variance model and the Finite Moment Log-Stable process. The calibration exercise shows that the CEV model is not able to produce enough slope over a short term because of its nature of pure diffusion. As a pure jump process, the FMLS process is able to match the slope of the short-term market data.

The paper is organized as follows. In Section 2, we define a few concepts and present our theoretical results. We describe the data and document the empirical evidence of the S\&P 500 index options in Section 3. In Section 4, we propose a new semi-analytical method to calibrate option-pricing models based on the quantified implied volatility smirk, and investigate the applicability of two option-pricing models. Finally, we conclude the paper in Section 5. 


\section{Quantify Implied Volatility Smirk}

Most of the existing research on the implied volatility smirk is qualitative in nature, partially because we lack appropriate quantities of data to describe the implied volatility - strike

price curve. For the convenience of quantifying an implied volatility smirk, we need to define a few concepts: implied forward price, moneyness, the implied volatility level, slope and curvature.

\subsection{Definitions}

The implied forward price, $F_{0}$, is determined based on the put-call parity from option prices with a certain maturity and a certain at-the-money strike. The at-the-money strike is the strike price at which the difference between the call and put prices is the smallest. With this implied forward price, one may verify with market data whether the put-call parity holds for options at other strikes.

The moneyness, $\xi$, is defined as the logarithm of the strike price, $K$, over the implied forward price, $F_{0}$, normalized by the standard deviation of the return on maturity, i.e.,

$$
\xi \equiv \frac{\ln \left(K / F_{0}\right)}{\bar{\sigma} \sqrt{\tau}}
$$

where $\bar{\sigma}$ denotes a measure of the average volatility of the underlying asset price. As pointed out by Carr and $\mathrm{Wu}$ (2003), the use of the constant $\bar{\sigma}$ in the denominator of (1) is an industry convention designed to allow comparisons across stocks. It also allows a simple interpretation of this moneyness measure as roughly the number of standard deviations that the log strike is away from the log forward price in the Black-Scholes model.

The implied volatility, $I V$, is computed by equating the Black-Scholes formula to the market price of an option. As documented by Foresi and Wu (2005), the implied volatility as a function of strike/moneyness for a certain maturity, is a negatively skewed curve for most of the index options. This phenomenon is often called the implied volatility smirk in the financial industry.

At a fixed maturity, we propose to use a second-order polynomial to describe the implied 
volatility - moneyness function, i.e.,

$$
I V(\xi)=\gamma_{0}\left(1+\gamma_{1} \xi+\gamma_{2} \xi^{2}\right)
$$

where the first parameter, $\gamma_{0}$, referred to as the level of the implied volatility smirk, is an estimate of the at-the-money implied volatility (ATMV), with at-the-money defined as $\xi=0$, i.e., $K=F_{0}$. The other two parameters, $\gamma_{1}$ and $\gamma_{2}$, are the slope and curvature of the implied volatility smirk.

\subsection{Theory}

With the implied volatility function specified in a parametric form (2), we are able to recover the risk-neutral probability analytically.

Proposition 1. The cumulative risk-neutral probability density function with a quadratic implied volatility is

$$
F\left(S, T ; F_{0}, 0\right)=N(-d)+n(d) \frac{\gamma_{0}}{\bar{\sigma}}\left[\gamma_{1}+2 \gamma_{2} \frac{\ln \left(S / F_{0}\right)}{\bar{\sigma} \sqrt{\tau}}\right], \quad \tau=T
$$

where $S$ is the underlying stock price at maturity $T, N(\cdot)$ is a cumulative standard normal distribution function, given by

$$
N(x)=\int_{-\infty}^{x} n(y) d y, \quad n(y)=\frac{1}{\sqrt{2 \pi}} e^{-y^{2} / 2},
$$

and

$$
d=-\frac{\ln \left(S / F_{0}\right)+\frac{1}{2} V^{2} \tau}{V \sqrt{\tau}}, \quad V=\gamma_{0}\left[1+\gamma_{1} \frac{\ln \left(S / F_{0}\right)}{\bar{\sigma} \sqrt{\tau}}+\gamma_{2}\left(\frac{\ln \left(S / F_{0}\right)}{\bar{\sigma} \sqrt{\tau}}\right)^{2}\right] .
$$

The recovered risk-neutral probability density function is

$$
\begin{aligned}
f\left(S, T ; F_{0}, 0\right)= & \frac{1}{S} \frac{n(d)}{V \sqrt{\tau}}\left[1+d \frac{\gamma_{0}}{\bar{\sigma}}\left(\gamma_{1}+2 \gamma_{2} \frac{\ln \left(S / F_{0}\right)}{\bar{\sigma} \sqrt{\tau}}\right)\right] \times \\
& {\left[1+(d+V \sqrt{\tau}) \frac{\gamma_{0}}{\bar{\sigma}}\left(\gamma_{1}+2 \gamma_{2} \frac{\ln \left(S / F_{0}\right)}{\bar{\sigma} \sqrt{\tau}}\right)\right]+\frac{n(d)}{S} \frac{2 \gamma_{0}}{\bar{\sigma}^{2} \sqrt{\tau}} \gamma_{2} . }
\end{aligned}
$$

Proof. See Appendix A. 
Remark 1. Shimko (1993) proposes fitting a smooth curve to an implied volatility function and recovers the risk-neutral density by using Breeden and Litzenberger (1978) formula. Rubinstein (1994), and Jackwerth and Rubinstein (1996) use a numerical optimization technique to recover the risk-neutral density. Aït-Sahalia and Lo (1998) constructs a nonparametric estimation for the risk-neutral density and derives its asymptotic sampling theory. We fit the implied volatility function by a quadratic function and present an explicit and intuitive formula for the risk-neutral density. The goodness-to-fit will be verified empirically with the S\&P 500 index options in the next section.

The proposition relates the coefficients in the implied volatility function to the riskneutral probability. If both the slope and the curvature vanish, the risk-neutral probability is reduced to a classical Black-Scholes lognormal distribution. The impact of the slope and curvature on the risk-neutral distribution, described analytically by the two formulas (3, 5), is depicted in Figure 1. The figure is for a sample of the level, slope and curvature obtained in the next section by fitting the market data on November 4, 2003. One may observe that the negative slope in the implied volatility makes the probability density skewed to the left. The positive curvature in the implied volatility makes the probability density leptokurtic, that is, it has a higher peak and two heavier tails than those of the lognormal distribution.

With some analysis, we may further obtain the relation between the implied volatility level, slope and curvature and the risk-neutral standard deviation, skewness and excess kurtosis.

Proposition 2. The level, slope and curvature of the implied volatility smirk, $\left(\gamma_{0}, \gamma_{1}\right.$, $\left.\gamma_{2}\right)$, and the risk-neutral standard deviation, skewness and excess kurtosis, $\left(\sigma, \lambda_{1}, \lambda_{2}\right)$ are approximately related by following equations

$$
1-2 N(d) \approx\left[N\left(d_{1}\right)-N\left(d_{2}\right)\right]\left[1+\frac{\lambda_{1}}{3 !}(\sigma \sqrt{\tau})^{3}+\frac{\lambda_{2}}{4 !}(\sigma \sqrt{\tau})^{4}\right]+\left(\frac{\lambda_{1}}{3 !} A+\frac{\lambda_{2}}{4 !} B\right) \sigma \sqrt{\tau},
$$




$$
\begin{aligned}
& N(-d)+n(d) \frac{\gamma_{0}}{\bar{\sigma}} \gamma_{1} \approx N\left(-d_{2}\right)-\left[\frac{\lambda_{1}}{3 !}\left(d_{2}^{2}-1\right)-\frac{\lambda_{2}}{4 !}\left(d_{2}^{3}-3 d_{2}\right)\right] n\left(d_{2}\right) \\
& \frac{1}{\gamma_{0} \sqrt{\tau}}\left(1-d^{2} \frac{\gamma_{0}^{2}}{\bar{\sigma}^{2}} \gamma_{1}^{2}+\frac{2 \gamma_{0}^{2}}{\bar{\sigma}^{2}} \gamma_{2}\right) n(d) \approx \frac{1}{\sigma \sqrt{\tau}}\left[1-\frac{\lambda_{1}}{3 !}\left(d_{2}^{3}-3 d_{2}\right)+\frac{\lambda_{2}}{4 !}\left(d_{2}^{4}-6 d_{2}^{2}+3\right)\right] n\left(d_{2}\right),
\end{aligned}
$$

where

$$
\begin{aligned}
d & =-\frac{1}{2} \gamma_{0} \sqrt{\tau}, \quad d_{2}=-\frac{1}{2} \sigma \sqrt{\tau}+\frac{\mu \sqrt{\tau}}{\sigma}, \quad d_{1}=d_{2}+\sigma \sqrt{\tau} \\
\mu & =-\frac{1}{\tau} \ln \left[1+\frac{\lambda_{1}}{3 !}(\sigma \sqrt{\tau})^{3}+\frac{\lambda_{2}}{4 !}(\sigma \sqrt{\tau})^{4}\right] \\
A & =-n^{\prime}\left(-d_{2}\right)+\sigma \sqrt{\tau} n\left(-d_{2}\right)+\sigma^{2} \tau N\left(d_{2}\right)=-\left(d_{2}-\sigma \sqrt{\tau}\right) n\left(d_{2}\right)+\sigma^{2} \tau N\left(d_{2}\right), \\
B & =n^{\prime \prime}\left(-d_{2}\right)-\sigma \sqrt{\tau} n^{\prime}\left(-d_{2}\right)+\sigma^{2} \tau n\left(-d_{2}\right)+(\sigma \sqrt{\tau})^{3} N\left(d_{2}\right) \\
& =-\left(1-d_{2}^{2}+\sigma \sqrt{\tau} d_{2}-\sigma^{2} \tau\right) n\left(d_{2}\right)+(\sigma \sqrt{\tau})^{3} N\left(d_{2}\right) .
\end{aligned}
$$

The result is obtained asymptotically by matching two option-pricing formulas at-the-money, and their first- and second-order sensitivities with respect to the strike at-the-money. The two option-pricing formulas are obtained with two different approaches: the Black-Scholes formula with a known implied volatility function, and risk-neutral valuation formula with moment expansion method.

Proof. See Appendix B.

The left-hand side of formula (6) depends only on the implied volatility level, slope and curvature, while the right-hand side depends only on the risk-neutral standard deviation, skewness and excess kurtosis. Given one set of the two sets of parameters, we may solve the three equations for the other set.

When deriving the relation, we price options by using two different approaches. One is the Black-Scholes formula with a known implied volatility function; the other one is the risk-neutral valuation formula that uses the Edgeworth expansion of the probability density with known skewness and excess kurtosis. The three relations in Proposition 2 are derived under the three matching conditions that two option-pricing formulas give the same values for the at-the-money option, the first- and the second-order sensitivities of the option price with respect to the strike price. The three financially meaningful matching 
conditions essentially reflect the industry practice.

In practice, for example, in the over-the-counter currency options market, quotes are in terms of the at-the-money implied volatility, the risk reversal and the butterfly spread. The risk reversal is a portfolio of two options: to long a call with a price, $c$, and to short a put with a price, $p$, both out-of-the-money. Its value at-the-money, i.e., $K=F_{0}$, is given by

$$
\begin{aligned}
c\left(F_{0}\right. & +\Delta K)-p\left(F_{0}-\Delta K\right)=c\left(F_{0}+\Delta K\right)-c\left(F_{0}-\Delta K\right)+\Delta K e^{-r T} \\
& =\left.2 \Delta K \frac{\partial c}{\partial K}\right|_{K=F_{0}}+\Delta K e^{-r T}+O\left[(\Delta K)^{2}\right] \\
& =2 \Delta K e^{-r T}\left[\left.F\left(S, T ; F_{0}, 0\right)\right|_{S=F_{0}}-\frac{1}{2}\right]+O\left[(\Delta K)^{2}\right],
\end{aligned}
$$

where $\Delta K$ denotes a small change in the strike price and the put-call parity is used in the first equality. The notation, $O\left[(\Delta K)^{2}\right]$ represents the terms that are of the order of $(\Delta K)^{2}$. The risk reversal is used to trade the first-order sensitivity of the option price with respect to the strike price, or the cumulative risk-neutral probability at-the-money shifted by $1 / 2$.

The butterfly spread is a portfolio of three options: to long two calls, one in-the-money and one out-of-the-money, and to short two at-the-money calls. Its value at-the-money is given by

$$
\begin{aligned}
c\left(F_{0}\right. & +\Delta K)-2 c\left(F_{0}\right)+c\left(F_{0}-\Delta K\right)=\left.2(\Delta K)^{2} \frac{\partial^{2} c}{\partial K^{2}}\right|_{K=F_{0}}+O\left[(\Delta K)^{4}\right] \\
& =\left.(\Delta K)^{2} e^{-r T} f\left(S, T ; F_{0}, 0\right)\right|_{S=F_{0}}+O\left[(\Delta K)^{4}\right] .
\end{aligned}
$$

The butterfly spread is used to trade the second-order sensitivity of the option price with respect to the strike price, or the risk-neutral probability density at-the-money.

If we use the market quotations of the at-the-money option, the risk reversal and the butterfly spread to determine the level, slope and curvature of the implied volatility smirk, then the risk-neutral standard deviation, skewness and excess kurtosis computed from Proposition 2 will preserve the market quotes of these option portfolios.

To present the intuition, we further simplify the result in Proposition 2. 
Proposition 3. The level, slope and curvature of the implied volatility smirk, $\left(\gamma_{0}, \gamma_{1}\right.$, $\left.\gamma_{2}\right)$, and the risk-neutral standard deviation, skewness and excess kurtosis, $\left(\sigma, \lambda_{1}, \lambda_{2}\right)$ are related by the following asymptotic expansion formulas:

$$
\begin{aligned}
\gamma_{0} & =\left(1-\frac{\lambda_{2}}{24}\right) \sigma+\frac{\lambda_{1}}{4} \sigma^{2} \sqrt{\tau}+O\left(\sigma^{3} \tau\right), \\
\gamma_{1} & =\frac{\lambda_{1}}{6\left(1-\frac{\lambda_{2}}{24}\right)} \frac{\bar{\sigma}}{\sigma}+\frac{\lambda_{2}\left(1-\frac{\lambda_{2}}{24}\right)-\frac{\lambda_{1}^{2}}{2}}{12\left(1-\frac{\lambda_{2}}{24}\right)^{2}} \bar{\sigma} \sqrt{\tau}+O(\sigma \bar{\sigma} \sqrt{\tau}), \\
\gamma_{2} & =\frac{\lambda_{2}}{24} \frac{\bar{\sigma}^{2}}{\sigma^{2}} \frac{1-\frac{\lambda_{2}}{16}}{\left(1-\frac{\lambda_{2}}{24}\right)^{2}}+\frac{\lambda_{1} \lambda_{2}}{96} \frac{\bar{\sigma}^{2} \sqrt{\tau}}{\sigma} \frac{1-\frac{\lambda_{2}}{48}}{\left(1-\frac{\lambda_{2}}{24}\right)^{3}}+O\left(\bar{\sigma}^{2} \sqrt{\tau}\right) .
\end{aligned}
$$

Ignoring the second and higher-order terms and taking $\bar{\sigma}=\gamma_{0}$, we have following leadingorder result:

$$
\gamma_{0} \approx\left(1-\frac{\lambda_{2}}{24}\right) \sigma, \quad \gamma_{1} \approx \frac{1}{6} \lambda_{1}, \quad \gamma_{2} \approx \frac{1}{24} \lambda_{2}\left(1-\frac{\lambda_{2}}{16}\right)
$$

If we further assume that $\frac{\lambda_{2}}{16} \ll 1$, then we obtain the following simple result:

$$
\gamma_{0} \approx\left(1-\frac{\lambda_{2}}{24}\right) \sigma, \quad \gamma_{1} \approx \frac{1}{6} \lambda_{1}, \quad \gamma_{2} \approx \frac{1}{24} \lambda_{2}
$$

as a rule of thumb.

Proof. By expanding both sides of formula (6) for a small volatility, we may obtain the result by using some algebra.

Remark 2. The leading order result in equation (9) was presented in Backus et al. (1997), and Bouchaud and Potters (2003), see also, Potters, Cont and Bouchaud (1998).

The result in equation (9) is quite intuitive. It says that

The at-the-money implied volatility is equal to the risk-neutral standard deviation with a small correction from the excess kurtosis, the slope of the implied volatility smirk is one-sixth of the risk-neutral skewness, and the curvature of the implied volatility smirk is one-24th of the risk-neutral excess kurtosis. 
The result is approximately correct in the sense that all of these values are much smaller than unity. In the next section, we show empirically that the condition is valid for the $\mathrm{S} \& \mathrm{P}$ 500 index options.

\section{Data and Evidence}

Following Duffie, Pan and Singleton (2000), Aït-Sahalia, Wang and Yared (2001), and Eraker (2004), we use daily closing prices of the S\&P 500 (SPX) index options to study the phenomenon of the implied volatility smirk. The data from three days: October 30, November 4 , and November $12,2003^{5}$, are downloaded from the website of the Chicago Board Options Exchange (CBOE). The historical data from September 1998 to October 1999 are provided by a commercial data supplier. The data of the risk-free rate are downloaded from the website of United States Department of Treasury.

\subsection{Data Processing and Fitting Methodology}

We use the data from November 4, 2003 in Table 1 to demonstrate the procedure of processing the data and producing the implied volatility function. All the options are sorted in ascending order by the strike price ranging between 850 and 1125 . The lowest strike price is selected from the first out-of-the-money put with a non-zero bid price. The highest strike is selected from the first out-of-the-money call with a non-zero bid price. ${ }^{6}$ There is a notable difference between the last sale price and mid-value of the bid and the ask. Some of the last sale prices even fall outside of the range between the bid and the ask. This is because the last sale happened some time before the market closed. Since it is difficult to determine the time instant of the last sale, we do not use the last sale price as a proxy for the daily closing price. We use the mid-value of the closing bid and the closing ask instead. The at-the-money strike is $K=1055$, at which the difference between the call

\footnotetext{
${ }^{5}$ These dates were randomly picked when we prepared the first draft of the paper.

${ }^{6}$ We follow the practice set up by the CBOE in computing the new volatility index, VIX. The definition of the VIX and the methodology of computing it from options prices are clearly described in the CBOE white paper, available at: http://www.cboe.com/micro/vix/vixwhite.pdf. The CBOE started trading VIX futures on March 26, 2004, and VIX options on February 24, 2006. Carr and Wu (2006), Zhang and Zhu (2006), and Zhu and Zhang (2007) study the price of VIX futures and their relationship with the underlying S\&P 500 index options.
} 
and the put prices is the smallest. The implied forward price is determined based on the at-the-money option prices

$$
\begin{aligned}
F_{0} & =\text { Strike price }+e^{r \tau} \times(\text { Call price }- \text { Put price }) \\
& =1055+e^{0.9743 \% \times 17 / 365}(11.9-14.2)=1052.70
\end{aligned}
$$

where the risk-free rate, $r$, is determined by the U.S. treasury yield curve rates on November 4, 2003, provided in Table 2. Since the yield curve starts from the one month rate and the term of the options is only 17 days, an extrapolation technique is used to compute the rate for 17 days, i.e.,

$r=r_{1 \mathrm{mth}}-\left(r_{3 \mathrm{mth}}-r_{1 \mathrm{mth}}\right) \times \frac{30-17}{61}=0.97 \%-(0.95 \%-0.97 \%) \times \frac{30-17}{61}=0.9743 \%$.

The forward index level, $F_{0}=1052.70$, is slightly smaller than the closing index, $S_{0}=$ 1053.25. This means that the implied dividend yield, $q=r-\ln \left(F_{0} / S_{0}\right)$, is slightly larger than the risk-free rate, $r$.

With the implied forward index level, we now verify that the put-call parity holds for the market prices of options with strikes other than at-the-money. The time value of an option (call or put) is defined to be the difference between the option price and its intrinsic value as follows

$$
\begin{aligned}
& c_{t v}(K)=c_{0}(K)-e^{-r \tau} \max \left(F_{0}-K, 0\right), \\
& p_{t v}(K)=p_{0}(K)-e^{-r \tau} \max \left(K-F_{0}, 0\right) .
\end{aligned}
$$

If the put-call parity, $c_{0}(K)-p_{0}(K)=\left(F_{0}-K\right) e^{-r \tau}$, is true, then the time values of the call and the put must be the same, i.e., $c_{t v}(K)=p_{t v}(K)$. Figure 2 shows the computed time values of SPX index options on November 4, 2003 for both calls and puts that mature on November 17, 2003. The two time value functions from the calls and puts almost collapse on each other. This means that the put-call parity is valid for the market prices of options with different strikes on the day.

Since the put-call parity holds, the market data of either the call or the put gives the same value of the implied volatility. Following Carr and Wu (2003) and the practice set 
up by the CBOE in computing the new VIX index, we use out-of-the-money options to compute the implied volatilities for different strikes. We choose the put option for the strike price that is below the forward index, $K<F_{0}$, and choose the call option for the strike price that is above forward index, $K>F_{0}$. The exclusive use of out-of-the-money options is an industry convention that arises from their greater liquidity and model sensitivity than their in-the-money counterparts.

A sample of implied volatility smirk data on November 4, 2003 for SPX options that mature on November 21, 2003 is given in Table 3. The table contains the information on the market prices and trading volumes of all the out-of-the-money calls and puts with non-zero bid. When computing the moneyness, we use the CBOE volatility index, VIX, as a proxy of the average volatility. We invert the Black-Scholes formula to obtain the implied volatility. We then use a quadratic function to fit the implied volatility data by minimizing the volume-weighted mean squared error

$$
\frac{\sum_{\xi} \text { Volume } \times\left[I V_{\text {market }}-I V(\xi)\right]^{2}}{\sum_{\xi} \text { Volume }}
$$

and obtain

$$
I V(\xi)=0.1447-0.0189 \xi+0.00595 \xi^{2}=0.1447\left(1-0.1308 \xi+0.0411 \xi^{2}\right),
$$

or the smirk parameters of the day,

$$
\left(\gamma_{0}, \gamma_{1}, \gamma_{2}\right)=(0.1447,-0.1308,0.0411)
$$

When we do the fitting, we force the curve to pass through the point at the money. In other words, the point $(\xi, I V)=(0,0.1447)$ is given by the market data. Therefore, the fitted implied volatility smirk gives exactly the same price as the market price for an option at-the-money. There is no arbitrage between the model price and the market price for an option at-the-money.

The implied volatility smirk is shown graphically in Figure 3. The dots are the implied volatilities from the market prices of the out-of-the-money calls and puts with different strikes. The solid line is a fitted quadratic curve. One may observe that the quadratic 
function approximates the implied volatility smirk very well. This is confirmed by the fitted errors provided in Table 3. The root of the volume-weighted mean squared error is only 0.0023 , which is about $1.5 \%$ of the average volatility, VIX $=0.1655$, on the day.

We now numerically study the impact of the slope and curvature of the implied volatility on the option price. For one set of options with the same maturity, one may use three different functions of moneyness to describe the implied volatility,

$$
\begin{array}{ll}
\text { flat } & I V=\gamma_{0}, \\
\text { skewed } & I V=\gamma_{0}\left(1+\gamma_{1} \xi\right), \\
\text { smirked } & I V=\gamma_{0}\left(1+\gamma_{1} \xi+\gamma_{2} \xi^{2}\right) .
\end{array}
$$

The flat implied volatility function is a constant that corresponds to the Black-Scholes model with the volatility equal to the ATMV. The skewed one is a linear function that passes through the point at-the-money and incorporates the slope. The smirked one is a quadratic function with both the slope and curvature. It becomes a pure smile if the slope, $\gamma_{1}$, is zero. The last case is thus the most general one. Figure 4 shows the difference between three implied volatility functions with the market implied volatilities as a reference. The market implied volatilities, shown as black dots in the figure, is the data from November 4, 2003 for the November SPX options.

Table 4 shows the option prices computed by using the Black-Scholes formula with flat, skewed, and smirked volatility functions. The root of the volume weighted mean squared error is 78 cents for the flat implied volatility function (or the Black-Scholes constant volatility case), 31 cents for the skewed one, and 12 cents for the smirked one. The slope reduces the error by more than $60 \%$, and the curvature reduces the remaining error by another $60 \%$. With both the slope and the curvature, the final error is only 12 cents, which is smaller than the smallest bid-ask spread. Within all the traded options listed in Table 1, the SPX call with a strike of 1125 has the smallest bid-ask spread of 15 cents. The smirked implied volatility function is therefore good enough to capture the trend of the implied volatility - moneyness curve with the root of volume weighted mean squared error smaller than the smallest bid-ask spread. 
Figure 5 shows the option price and the price error as functions of the strike for different implied volatility functions, together with the trading volumes. It is quite impressive that the price given by the Black-Scholes formula with a smirked implied volatility function is so close to the market prices of the traded options.

With the level, slope and curvature of the implied volatility smirk already determined, $\left(\gamma_{0}, \gamma_{1}, \gamma_{2}\right)=(0.1447,-0.1308,0.0411)$, solving the three equations in (6) numerically gives the three risk-neutral cumulants:

$$
\begin{aligned}
\text { standard deviation } & \sigma=0.1506, \\
\text { skewness } & \lambda_{1}=-0.6992, \\
\text { excess kurtosis } & \lambda_{2}=0.8065 .
\end{aligned}
$$

To verify Proposition 3, substituting these risk-neutral cumulants into the right-hand-side of the three equations in (7), by taking the first terms in each equation, i.e., equation (8), we have the approximate values of the smirk parameters, i.e., the level, slope and curvature,

$$
\left(\gamma_{0}, \gamma_{1}, \gamma_{2}\right) \approx(0.1455,-0.1325,0.04126)
$$

By taking the first two terms in each equation, we obtain the set of smirk parameters as

$$
\left(\gamma_{0}, \gamma_{1}, \gamma_{2}\right) \approx(0.1447,-0.1308,0.0410)
$$

which is identical to the given set of values except the tiny difference in the curvature (0.0410 vs. 0.0411$)$.

This numerical exercise shows that the typical smirk parameters computed by using the first two terms of the three equations in (7) in Proposition 3 are almost identical to those given by three equations in (6) in Proposition 2.

\subsection{Empirical Evidence}

In the same way, we process the option data for November 4, 2003 for all available maturities, Nov-21-03, Dec-19-03, Jan-16-04, Mar-19-04, Jun-18-04, Sep-17-04, Dec-17-04, and 
Jun-17-05, with times to maturity of $17,45,73,136,227,318,409$, and 591 days, respectively. Shown in Figure 6 are the implied volatility curves together with the bar charts of trading volumes.

The numerical values of the term structure of the smirk parameters ${ }^{7}$ are listed in Table 5 together with the term structures of interest rates and trading volumes. The first and the second nearest options are heavily traded. They occupy $80 \%$ of the total trading volume. The term structure of the interest rate is upward sloping. The term structure of the ATMV is slightly upward sloping, ranging from $14 \%$ to $18 \%$. The term structure of the slope is quite flat with a value near -0.17 . The implied volatility smirk does not flatten out as maturity increases up to almost two years. This pattern has been documented qualitatively and explained with a Finite Moment Log Stable process by Carr and Wu (2003). The term structure of the curvature is oscillating between 0 and 0.04 .

To intuit the evolution of the term structure of the smirk parameters, we processed the market data for three different days, October 30, 2003, November 4, 2003 and November 12, 2003. The final numerical values of the term structures are listed in Table 6. They are presented graphically in Figure 7. In general, the term structures are stable with longer maturities. The term structure of the ATMV follows a more regular pattern than the other two term structures. This means that investors have a better understanding of the ATMV than the slope and curvature, probably because the latter two quantities have never been explicitly defined and documented in the literature.

In order to gain an idea about the time-change dynamics of the implied volatility smirk, we study the time series from September 25, 1998 to September 3, 1999 of market prices of SPX options with the same maturity date, September 16, 1999. After determining the level, slope and curvature of the implied volatility smirk for each day, we present the time series of the three parameters in Figure 8. The dynamics of the ATMV seems to be a mean-reverting process. The implied volatility slope increases from -0.35 to -0.2 as the time to maturity becomes shorter. The implied volatility curvature seems to be more noisy

\footnotetext{
${ }^{7}$ The term structure of implied volatility has been studied by Heynen, Kemna and Vorst (1994), Xu and Taylor (1994), Zhu and Avellaneda (1997), and Das and Sundaram (1999).
} 
than the other two parameters. The dynamics of the implied volatility slope and curvature basically describe the dynamics of risk-neutral skewness and excess kurtosis. Their relation with the subsequent realized statistical skewness and excess kurtosis is an interesting topic for further research ${ }^{8}$.

\section{Model calibration based on the implied volatility smirk}

Option pricing models are developed with certain numbers of embedded parameters, which are usually determined from market information by following one of two approaches. The first one is to estimate the parameters by using historical data of underlying stock prices. The problem with this approach is that the historical data may not reflect information from stock returns in the future. Practitioners often use a second approach to calibrate the parameters from the current market prices of liquidly-traded options.

Calibrating an option pricing model is a difficult task. It is commonly recognized that one should calibrate a model at least on a daily basis to include the latest information from the financial markets. At the end of a trading day, we have a closing price for each option with different strikes and maturities. Their trading volumes are different. Some of them, for example, the ones with the first and the second nearest maturities, are liquid. Others are not. The price of illiquid options does not tell us much information about the financial market.

One way to calibrate the option pricing model is to minimize the sum of the squared errors of all available options with different strikes and maturities. The liquidity effect can be accounted for by the volume weighting in the least squares approach. The procedure, initiated by Bakshi, Cao and Chen (1997), has become a standard in the empirical study of model performance (see, e.g., Carr et al (2003) and Huang and Wu (2004)). But this method suffers from two problems. First, the nonlinear least squares model is well known to be unstable, especially with a large set of parameters, such as the ten parameters in a

\footnotetext{
${ }^{8}$ For example, Navatte and Villa (2000) studies the information content of implied volatility, skewness and kurtosis by using long-term CAC 40 options.
} 
time-changed Lévy process in Huang and Wu (2004). The solution obtained with some standard commercial packages may not be unique. For the case when the solution is not unique, the values of the parameters are not meaningful. Second, the procedure is purely numerical. It is not easy to monitor and to compare the pricing errors of options with different maturities.

We propose a new maturity-based and liquidity-based calibration. The idea is to use the distilled information of the term structure of smirk parameters. Since we have already considered the liquidity issue in fitting the value of these parameters, all we need to do is to force the term structure of the smirk parameters implied from an option pricing model to pass through the points of the market's term structures, from the first nearest term ${ }^{9}$ to the second nearest one and so on.

The proposed method has two advantages: First, it is semi-analytical. This allows us to determine $n$ model parameters by solving $n$ equations. The problem of finding the root of $n$ equations is easier to solve than the minimization problem with $n$ unknowns. Second, the calibration procedure is more transparent. By comparing the market and model term structures of the smirk level, the slope and the curvature, we are able to monitor the performance of the calibration for each maturity.

For example, in the Black-Scholes (1973) model, the stock price is modelled as

$$
d S_{t}=(r-q) S_{t} d t+\sigma S_{t} d B_{t}
$$

where $r$ is the risk-free rate, $q$ is the continuous dividend yield, and $B_{t}$ is the standard Brownian motion. It has only one parameter, i.e., volatility, $\sigma$. One may simply calibrate the model by using ATMV, $\gamma_{0}$, for the volatility, $\sigma$. In fact, this is a classical way of using the Black-Scholes model.

In the following, we present the calibration of two parsimonious models and study the term structure of the smirk parameters implied in the calibrated option pricing models.

\footnotetext{
${ }^{9}$ Following the convention set up by CBOE in computing the new VIX index, with 8 days left to expiration, we roll to the second contract month in order to minimize pricing anomalies that might occur close to expiration.
} 


\subsection{The Constant Elasticity of Variance (CEV) Model}

In a risk-neutral world, the price of a stock, $S_{t}$, is assumed to follow a diffusion process

$$
d S_{t}=(r-q) S_{t} d t+\sigma S_{t}^{\alpha} d B_{t}
$$

where $\sigma$ and $\alpha$ are constants. The variance in stock returns is given by $v_{t}=\sigma^{2} S_{t}^{2 \alpha-2}$, and the elasticity of variance, defined by $\frac{S_{t}}{v_{t}} \frac{\partial v_{t}}{\partial S_{t}}$, is therefore a constant, $2 \alpha-2$.

Cox (1975) finds that, with a power transformation, $X_{t}=S_{t}^{2(1-\alpha)}$, the CEV process can be transformed into a square-root process:

$$
d X_{t}=\left[(1-\alpha)(1-2 \alpha) \sigma^{2}+2(1-\alpha)(r-q) X_{t}\right] d t+2(1-\alpha) \sigma \sqrt{X_{t}} d B_{t} .
$$

Feller (1951) studies the transition probability of the square-root process with a Laplace transformation approach and provides an analytical formula for the density function. The price of a European call option can therefore be determined with a risk-neutral valuation formula. The term structure of the smirk parameters implied in the CEV model is given in the following proposition.

Proposition 4. For $0<\alpha<1$, the smirk parameters, $\left(\gamma_{0}, \gamma_{1}, \gamma_{2}\right)$, implied in the $C E V$ model can be determined by

$$
\begin{aligned}
& 1-2 N(d)=Q\left(2 x ; 2+\frac{1}{1-\alpha}, 2 x\right)+Q\left(2 x ; \frac{1}{1-\alpha}, 2 x\right)-1, \\
& N(-d)+n(d) \frac{\gamma_{0}}{\bar{\sigma}} \gamma_{1}=Q\left(2 x ; \frac{1}{1-\alpha}, 2 x\right), \\
& \frac{1}{\gamma_{0} \sqrt{\tau}}\left(1-d^{2} \frac{\gamma_{0}^{2}}{\bar{\sigma}^{2}} \gamma_{1}^{2}+\frac{2 \gamma_{0}^{2}}{\bar{\sigma}^{2}} \gamma_{2}\right) n(d)=4(1-\alpha) x p\left(2 x ; 2-\frac{1}{1-\alpha}, 2 x\right),
\end{aligned}
$$

where $p(z ; n, \lambda)$ is the non-central chi-square distribution function with $n$ degrees of freedom and non-centrality parameter $\lambda, Q(z ; n, \lambda)$ is the complementary non-central chi-square distribution, and

$$
d=-\frac{1}{2} \gamma_{0} \sqrt{\tau}, \quad x=\frac{(r-q) F_{0}^{2(1-\alpha)}}{(1-\alpha) \sigma^{2}\left[e^{2(1-\alpha)(r-q) \tau}-1\right]} .
$$

Proof. See Appendix C. 
The CEV model has two parameters, volatility, $\sigma$, and the elasticity constant, $\alpha$. The exercise of calibrating the CEV model requires using the ATMV, $\gamma_{0}$, and slope, $\gamma_{1}$, of the nearest term (the most liquid one) of the implied volatility smirk to determine these two constants. A general intuition is that the slope is negative for $0 \leq \alpha<1$, zero for $\alpha=1$ (the Black-Scholes case) and positive for $\alpha>1$. The smaller the value of $\alpha$ between 0 and 1, the larger the absolute value of the slope.

For the data from November 4, 2003 processed in the last section, $\gamma_{0}=0.1447, \gamma_{1}=$ -0.1308 , we find that the largest negative slope that the CEV model can produce is $\gamma_{1}=$ -0.0179 for $\alpha=0$, which is much smaller than the required value from the market data. The result indicates that the $\mathrm{CEV}$ model cannot produce enough negative risk-neutral skewness in the short term, simply because the model is based on a pure diffusion. One needs negative jumps in the underlying process to accomplish the task. With the best set of calibrated parameters, $(\sigma, \alpha)=(152.36,0)$, we compute the term structure of the smirk parameters implied in the CEV model from Proposition 4. The results are given in Table 7 numerically and shown in Figure 9 graphically together with results from the market data. An interesting observation is that the CEV model is able to produce a large negative skewness in the long term.

\subsection{The Finite Moment Log Stable (FMLS) model}

Recently, Carr and Wu (2003) proposed a Finite Moment Log $\alpha$-Stable (FMLS) process for stock prices,

$$
d S_{t}=(r-q) S_{t} d t+\sigma S_{t} d L_{t}^{\alpha,-1}
$$

where $\sigma$ is a constant, $d L_{t}^{\alpha,-1}$ has an $\alpha$-stable distribution with zero drift, dispersion of $d t^{1 / \alpha}$, and a skew parameter of $-1: L_{\alpha}\left(0, d t^{1 / \alpha},-1\right)$. The $\alpha$-stable process is a pure jump Lévy process when $0<\alpha<2$. When $\alpha=2$, the $\alpha$-stable process degenerates to a standard Brownian motion multiplied by $\sqrt{2}$, i.e., $L_{t}^{2, \beta}=\sqrt{2} B_{t}$.

The stock price at a future time, $T$, is then written explicitly as

$$
S_{T}=S_{t} e^{(r-q+\mu) \tau+\sigma L_{\tau}^{\alpha,-1}}, \quad \tau=T-t
$$




$$
\ln \left(S_{T} / S_{t}\right) \sim L_{\alpha}\left((r-q+\mu) \tau, \sigma \tau^{1 / \alpha},-1\right)
$$

where the convexity adjustment term is given by $\mu=\sigma^{\alpha} \sec \frac{\pi \alpha}{2}$ to ensure the martingale condition, $E_{t}^{Q}\left[S_{T}\right]=S_{t} e^{(r-q) \tau}$. The characteristic function of $\ln \left(S_{T} / S_{t}\right)$ is given by (Carr and $\mathrm{Wu} 2003)$

$$
f(\tau ; \phi)=E_{t}^{Q}\left[e^{i \phi \ln \left(S_{T} / S_{t}\right)}\right]=e^{i \phi(r-q) \tau+\left(i \phi-(i \phi)^{\alpha}\right) \mu \tau} .
$$

The term structure of the smirk parameters implied in the FMLS model is given in the following proposition.

Proposition 5. For $1<\alpha<2$, the smirk parameters, $\left(\gamma_{0}, \gamma_{1}, \gamma_{2}\right)$, implied in the FMLS process option pricing model can be determined by

$$
\begin{aligned}
& 1-2 N(d)=\frac{1}{\pi} \int_{0}^{\infty} \operatorname{Re}\left[\frac{e^{\left(i \phi+1-(i \phi+1)^{\alpha}\right) \mu \tau}-e^{\left(i \phi-(i \phi)^{\alpha}\right) \mu \tau}}{i \phi}\right] d \phi, \\
& N(-d)+n(d) \frac{\gamma_{0}}{\bar{\sigma}} \gamma_{1}=\frac{1}{2}-\frac{1}{\pi} \int_{0}^{\infty} \operatorname{Re}\left[\frac{e^{\left(i \phi-(i \phi)^{\alpha}\right) \mu \tau}}{i \phi}\right] d \phi, \\
& \frac{1}{\gamma_{0} \sqrt{\tau}}\left(1-d^{2} \frac{\gamma_{0}^{2}}{\bar{\sigma}^{2}} \gamma_{1}^{2}+\frac{2 \gamma_{0}^{2}}{\bar{\sigma}^{2}} \gamma_{2}\right) n(d)=\frac{1}{\pi} \int_{0}^{\infty} \operatorname{Re}\left[e^{\left(i \phi-(i \phi)^{\alpha}\right) \mu \tau}\right] d \phi,
\end{aligned}
$$

where $\mu=\sigma^{\alpha} \sec \frac{\pi \alpha}{2}$ is the convexity adjustment.

Proof. See Appendix D.

The model has only two parameters, $(\sigma, \alpha)$, as well. Similar to the CEV model, one should use the ATMV, $\gamma_{0}$, and slope, $\gamma_{1}$, of the implied volatility smirk of the nearest term to calibrate the model.

On November 4, 2003, the VIX index level was $\bar{\sigma}=0.1655$. The ATMV was $\gamma_{0}=0.1447$. The slope was $\gamma_{1}=-0.1308$. The time to maturity of the nearest term was $\tau=17 / 365$. Then, $d=-\frac{1}{2} \gamma_{0} \sqrt{\tau}=-0.0156141$. The task of calibrating the FMLS process option pricing model requires solving the following two equations

$$
\frac{1}{\pi} \int_{0}^{\infty} \operatorname{Re}\left[\frac{e^{\left(i \phi+1-(i \phi+1)^{\alpha}\right) \tau \sigma^{\alpha} \sec \frac{\pi \alpha}{2}}-e^{\left(i \phi-(i \phi)^{\alpha}\right) \tau \sigma^{\alpha} \sec \frac{\pi \alpha}{2}}}{i \phi}\right] d \phi=1-2 N(d)=0.0124577,
$$




$$
\frac{1}{2}-\frac{1}{\pi} \int_{0}^{\infty} \operatorname{Re}\left[\frac{e^{\left(i \phi-(i \phi)^{\alpha}\right) \tau \sigma^{\alpha} \sec \frac{\pi \alpha}{2}}}{i \phi}\right] d \phi=N(-d)+n(d) \frac{\gamma_{0}}{\bar{\sigma}} \gamma_{1}=0.460611
$$

for two unknowns, the volatility parameter, $\sigma$, and the tail index, $\alpha$. This task can be accomplished within a second by using three lines of Mathematica code. The result is $\sigma=0.1086, \alpha=1.8141$

With this set of calibrated parameters, we can compute the term structure of the smirk parameters implied in the FMLS process option pricing model from Proposition 5. The results are presented in Table 7 numerically and shown in Figure 9 graphically together with those from the CEV model and the market data. A direct comparison shows that the FMLS model is much better than the CEV model in terms of capturing the slope of the implied volatility smirk. This observation, which agrees with Carr and Wu (2003), indicates that the underlying process is indeed having jumps rather than a simple pure diffusion.

Other models, such as the jump-diffusion model of Merton (1976), the stochastic volatility model of Heston (1993), the variance Gamma process of Madan, Carr and Chang (1998), the affine jump diffusion model of Duffie, Pan and Singleton (2000), and the time-changed Lévy process of Carr et al. (2003), and Carr and Wu (2004) have more parameters. More points from the term structure of the smirk parameters are needed in calibration. The details of implementing our calibration procedure will be presented in a subsequent paper.

\section{Conclusion}

In this paper, we propose to use a quadratic function to quantify the shape (level, slope and curvature) of the implied volatility smirk. Then, we link the quadratic smirk shape to the risk-neutral return density and cumulants. We also show how such links can be used to calibrate and compare different models.

Empirical evidence from S\&P 500 index options shows that a quadratic function with both slope and curvature fits the implied volatility smirk very well. The volume-weighted error can be smaller than the smallest bid-ask spread of traded options. With these new 
quantities well defined, we are able to study the term structure, its evolution and the time-change dynamics of an implied volatility smirk.

Our research suggests that the three term structures of the at-the-money implied volatility, slope and curvature should be constructed on a daily basis. These term structures distilled from the current prices of options with different strikes and maturities provide a lot of information about an investor's fair expectations on the index return distribution over different terms in the future. They should therefore be used to calibrate option pricing models.

\section{A Proof of Proposition 1}

Since the price of a European call is given by the Black-Scholes formula with a quadratic implied volatility function, i.e.,

$$
c_{0}=F_{0} e^{-r \tau} N\left(d^{*}+I V \sqrt{\tau}\right)-K e^{-r \tau} N(d)
$$

where

$$
d^{*}=\frac{\ln \left(F_{0} / K\right)-\frac{1}{2} I V^{2} \tau}{I V \sqrt{\tau}}, \quad I V=\gamma_{0}\left(1+\gamma_{1} \xi+\gamma_{2} \xi^{2}\right), \quad \xi=\frac{\ln \left(K / F_{0}\right)}{\bar{\sigma} \sqrt{\tau}},
$$

then the risk-neutral probability can be recovered by Breeden and Litzenberger's (1978) formula,

$$
F\left(S, T ; F_{0}, 0\right)=1+\left.e^{r \tau} \frac{\partial c_{0}}{\partial K}\right|_{K=S}, \quad f\left(S, T ; F_{0}, 0\right)=\left.e^{r \tau} \frac{\partial^{2} c_{0}}{\partial K^{2}}\right|_{K=S} .
$$

With some algebra, one may obtain formulas $(3,5)$ in Proposition 1.

\section{B Proof of Proposition 2}

Suppose in a risk-neutral world, the underlying stock price at maturity, $S_{\tau}$, is modelled by

$$
S_{\tau}=F_{0} e^{\left(-\frac{1}{2} \sigma^{2}+\mu\right) \tau+\sigma \sqrt{\tau} y}
$$


where $\mu$ is the convexity adjustment, $y$ is a random number with mean zero, variance 1 , skewness ${ }^{10} \lambda_{1}$, and excess kurtosis $\lambda_{2}$. If $y$ is normally distributed, then $\mu=0$. The probability density of $y$ is given by the Edgeworth series expansion ${ }^{11}$

$$
f(y)=n(y)-\frac{\lambda_{1}}{3 !} \frac{d^{3} n(y)}{d y^{3}}+\frac{\lambda_{2}}{4 !} \frac{d^{4} n(y)}{d y^{4}}
$$

where $n(y)$ is the standard normal density function given by $(4)^{12}$.

The martingale condition in the risk-neutral probability measure requires that

$$
F_{0}=E_{0}^{Q}\left[S_{\tau}\right]=E_{0}^{Q}\left[F_{0} e^{\left(-\frac{1}{2} \sigma^{2}+\mu\right) \tau+\sigma \sqrt{\tau} y}\right], \quad \text { or } \quad E_{0}^{Q}\left[e^{\left(-\frac{1}{2} \sigma^{2}+\mu\right) \tau+\sigma \sqrt{\tau} y}\right]=1,
$$

which determines the convexity adjustment term,

$$
\mu=-\frac{1}{\tau} \ln \left[1+\frac{\lambda_{1}}{3 !}(\sigma \sqrt{\tau})^{3}+\frac{\lambda_{2}}{4 !}(\sigma \sqrt{\tau})^{4}\right]
$$

The price of a European call option can be computed with the Harrison and Kreps (1979), and Harrison and Pliska (1981) risk-neutral valuation formula,

$$
c_{0}^{*}=e^{-r \tau} E_{0}^{Q}\left[\max \left(S_{\tau}-K, 0\right)\right]=e^{-r \tau} \int_{-d_{2}}^{+\infty}\left(F_{0} e^{\left(-\frac{1}{2} \sigma^{2}+\mu\right) \tau+\sigma \sqrt{\tau} y}-K\right) f(y) d y,
$$

where

$$
d_{2}=\frac{\ln \left(F_{0} / K\right)+\left(-\frac{1}{2} \sigma^{2}+\mu\right) \tau}{\sigma \sqrt{\tau}} .
$$

With integration by parts, we have the following formula ${ }^{13}$

$$
\begin{aligned}
c_{0}^{*}= & {\left[F_{0} e^{-r \tau} N\left(d_{1}\right)-K e^{-r \tau} N\left(d_{2}\right)\right]\left[1+\frac{\lambda_{1}}{3 !}(\sigma \sqrt{\tau})^{3}+\frac{\lambda_{2}}{4 !}(\sigma \sqrt{\tau})^{4}\right] } \\
& +K e^{-r \tau}\left(\frac{\lambda_{1}}{3 !} A+\frac{\lambda_{2}}{4 !} B\right) \sigma \sqrt{\tau}
\end{aligned}
$$

${ }^{10}$ The skewness, $\lambda_{1}$, and excess kurtosis, $\lambda_{2}$, of a random number, $x$, are defined by $\lambda_{1}=\kappa_{3} / \kappa_{2}^{3 / 2}, \lambda_{2}=$ $\kappa_{4} / \kappa_{2}^{2}$, where $\kappa_{i}, i=1,2,3,4$ are the first four cumulants, given by $\kappa_{1}=E(x), \kappa_{2}=E\left(x-\kappa_{1}\right)^{2}=\operatorname{Var}(x)$, $\kappa_{3}=E\left(x-\kappa_{1}\right)^{3}, \kappa_{4}=E\left(x-\kappa_{1}\right)^{4}-3 \kappa_{2}^{2}$.

${ }^{11}$ An explanation of the Edgeworth series expansion method can be found in Chapter 3 (page 25) in Kolassa's (1997) book. A moment expansion approach to option pricing was studied by Airoldi (2005).

${ }^{12}$ Here we use Edgeworth series expansion to expand the unknown return distribution near a normal distribution. Jarrow and Rudd (1982) expand the unknown price distribution near a lognormal distribution and find a different option-pricing formula, see e.g., Corrado and $\mathrm{Su}$ (1997) for an empirical test of Jarrow and Rudd's (1982) model with SPX options. It seems to us that expanding the return distribution is more natural and consistent with the later advanced option-pricing models that model stock returns with a Lévy process or a time-changed Lévy process, see e.g., Carr and Wu (2004).

${ }^{13}$ The formula was presented by Backus et al. (1997) in the context of currency options. 
where $d_{1}=d_{2}+\sigma \sqrt{\tau}$, and

$$
\begin{aligned}
A & =-n^{\prime}\left(-d_{2}\right)+\sigma \sqrt{\tau} n\left(-d_{2}\right)+\sigma^{2} \tau N\left(d_{2}\right)=-\left(d_{2}-\sigma \sqrt{\tau}\right) n\left(d_{2}\right)+\sigma^{2} \tau N\left(d_{2}\right), \\
B & =n^{\prime \prime}\left(-d_{2}\right)-\sigma \sqrt{\tau} n^{\prime}\left(-d_{2}\right)+\sigma^{2} \tau n\left(-d_{2}\right)+(\sigma \sqrt{\tau})^{3} N\left(d_{2}\right) \\
& =-\left(1-d_{2}^{2}+\sigma \sqrt{\tau} d_{2}-\sigma^{2} \tau\right) n\left(d_{2}\right)+(\sigma \sqrt{\tau})^{3} N\left(d_{2}\right) .
\end{aligned}
$$

This call option pricing formula is exact under the assumption that the risk-neutral probability density is given by equation (23).

We now match the two option pricing formulas given by equations (21) and (24). Applying the three conditions at the money,

$$
\left.\left(c_{0}-c_{0}^{*}\right)\right|_{K=F_{0}}=0,\left.\quad \frac{\partial\left(c_{0}-c_{0}^{*}\right)}{\partial K}\right|_{K=F_{0}}=0,\left.\quad \frac{\partial^{2}\left(c_{0}-c_{0}^{*}\right)}{\partial K^{2}}\right|_{K=F_{0}}=0,
$$

gives the result in Proposition 2.

\section{Proof of Proposition 4}

In order to prove Proposition 4, we need the following known results.

Lemma 1. For $0<\alpha<1$, the conditional risk-neutral transition probability density function, $f\left(S_{T}, T ; S_{t}, t\right)$, is given by (Cox 1975)

$$
\begin{aligned}
f\left(S_{T}, T ; S_{t}, t\right) & =2(1-\alpha) k^{\frac{1}{2(1-\alpha)}}\left(x w^{1-4 \alpha}\right)^{\frac{1}{4(1-\alpha)}} e^{-x-w} I_{-\frac{1}{2(1-\alpha)}}(2 \sqrt{x w}) \\
& =4(1-\alpha) k^{\frac{1}{2(1-\alpha)}} w^{\frac{1-2 \alpha}{2(1-\alpha)}} p\left(2 w ; 2-\frac{1}{1-\alpha}, 2 x\right)
\end{aligned}
$$

where

$$
k=\frac{r-q}{(1-\alpha) \sigma^{2}\left[e^{2(1-\alpha)(r-q) \tau}-1\right]}, \quad x=k S_{t}^{2(1-\alpha)} e^{2(1-\alpha)(r-q) \tau}, \quad w=k S_{T}^{2(1-\alpha)},
$$

and $I_{\nu}(z)$ is the modified Bessel function ${ }^{14}$ of the first kind of order $\nu, p(z ; n, \lambda)$ is the prob-

\footnotetext{
${ }^{14}$ The modified Bessel function of the first kind of order, $\nu$, is a solution of the ordinary differential equation

$$
W^{\prime \prime}+\frac{1}{z} W^{\prime}-\left(1+\frac{\nu^{2}}{z^{2}}\right) W=0 .
$$
}

The function can be written in a series form as follows:

$$
I_{\nu}=\sum_{k=0}^{+\infty} \frac{1}{k ! \Gamma(\nu+k+1)}\left(\frac{z}{2}\right)^{2 k+\nu} .
$$


ability density function of a non-central chi-square distribution ${ }^{15}$ with $n$ degrees of freedom and non-centrality parameter $\lambda$.

The price of a European call option is given by ${ }^{16}$ (Cox 1975, Cox and Ross 1976, Schroder 1989)

$$
\begin{aligned}
c_{t} & =S_{t} e^{-q \tau} Q\left(2 y ; 2+\frac{1}{1-\alpha}, 2 x\right)-K e^{-r \tau} Q\left(2 y ; 2-\frac{1}{1-\alpha}, 2 x\right) \\
& =S_{t} e^{-q \tau} Q\left(2 y ; 2+\frac{1}{1-\alpha}, 2 x\right)-K e^{-r \tau}\left[1-Q\left(2 x ; \frac{1}{1-\alpha}, 2 y\right)\right],
\end{aligned}
$$

where $y=k K^{2(1-\alpha)}$, and $Q(z ; n, \lambda)$ is the complementary, non-central chi-square distribution function, defined by $Q(z ; n, \lambda)=\int_{z}^{+\infty} p(u ; n, \lambda) d u$.

For $\alpha>1$, the transition probability density function, $f\left(S_{T}, T ; S_{t}, t\right)$, is given by

$$
f\left(S_{T}, T ; S_{t}, t\right)=2(\alpha-1) k^{\frac{1}{2(1-\alpha)}}\left(x w^{1-4 \alpha}\right)^{\frac{1}{4(1-\alpha)}} e^{-x-w} I_{\frac{1}{2(\alpha-1)}}(2 \sqrt{x w})
$$

and the European call option pricing formula is

$$
c_{t}=S_{t} e^{-q \tau} Q\left(2 x ; \frac{1}{\alpha-1}, 2 y\right)-K e^{-r \tau}\left[1-Q\left(2 y ; 2+\frac{1}{\alpha-1}, 2 x\right)\right]
$$

(Emanuel and MacBeth 1982).

Matching the two option pricing formulas in (27) and (21) with three conditions in (25) yields Proposition 4.

\section{Proof of Proposition 5}

We need the following known results.

Lemma 2. If the characteristic function of $\ln \left(S_{T} / S_{t}\right)$ is denoted as $f(\tau ; \phi)=E_{t}^{Q}\left[e^{i \phi \ln \left(S_{T} / S_{t}\right)}\right]$, then the price of a European call option can be written as (Bakshi and Madan 2000)

$$
c_{t}=S_{t} e^{-q \tau} \Pi_{1}(t, \tau)-K e^{-r \tau} \Pi_{2}(t, \tau),
$$

\footnotetext{
${ }^{15}$ The probability density function of a non-central chi-square distribution can be written in terms of the modified Bessel function as follows (Johnson and Kotz 1970):$$
p(z ; n, \lambda)=\frac{1}{2}(z / \lambda)^{\frac{1}{4}(n-2)} e^{-\frac{1}{2}(z+\lambda)} I^{\frac{n-2}{2}}(\sqrt{\lambda z}) .
$$

${ }^{16}$ The complementary, non-central chi-square distribution function, $Q(z ; n, \lambda)$, satisfies following identity

$$
Q(z ; n, \lambda)+Q(\lambda ; 2-n, z)=1 .
$$
}


where

$$
\begin{aligned}
& \Pi_{1}(t, \tau)=\frac{1}{2}+\frac{1}{\pi} \int_{0}^{\infty} \operatorname{Re}\left[\frac{e^{-i \phi \ln \left(K / S_{t}\right)} \times f(\tau ; \phi-i)}{i \phi f(\tau ;-i)}\right] d \phi \\
& \Pi_{2}(t, \tau)=\frac{1}{2}+\frac{1}{\pi} \int_{0}^{\infty} \operatorname{Re}\left[\frac{e^{-i \phi \ln \left(K / S_{t}\right)} \times f(\tau ; \phi)}{i \phi}\right] d \phi .
\end{aligned}
$$

Applying the general formula to the FMLS process yields following result.

Lemma 3. If the underlying stock price is modelled by the FMLS process in equation (15), then the price of a European call option is given by

$$
c_{t}=F_{t} e^{-r \tau} \Pi_{1}(t, \tau)-K e^{-r \tau} \Pi_{2}(t, \tau),
$$

where

$$
\begin{aligned}
\Pi_{1}(t, \tau) & =\frac{1}{2}+\frac{1}{\pi} \int_{0}^{\infty} \operatorname{Re}\left[\frac{e^{-i \phi \ln \left(K / F_{t}\right)+\left(i \phi+1-(i \phi+1)^{\alpha}\right) \mu \tau}}{i \phi}\right] d \phi, \\
\Pi_{2}(t, \tau) & =\frac{1}{2}+\frac{1}{\pi} \int_{0}^{\infty} \operatorname{Re}\left[\frac{e^{-i \phi \ln \left(K / F_{t}\right)+\left(i \phi-(i \phi)^{\alpha}\right) \mu \tau}}{i \phi}\right] d \phi,
\end{aligned}
$$

where $F_{t}=S_{t} e^{(r-q) \tau}$ is the current froward price, and $\mu=\sigma^{\alpha} \sec \frac{\pi \alpha}{2}$ is the convexity adjustment.

Matching the two option pricing formulas in (30) and (21) with three conditions in (25) yields Proposition 5.

\section{References}

[1] Airoldi M 2005 A moment expansion approach to option pricing Quant. Finance 5 89-104

[2] Aït-Sahalia Y and Lo A W 1998 Nonparametric estimation of state-price densities implicit in financial asset prices J. Finance 53 499-547

[3] Aït-Sahalia Y, Wang Y and Yared F 2001 Do options markets correctly assess the probabilities of movement in the underlying asset? J. Econometrics 102 67-110 
[4] Backus D, Foresi S, Li K and Wu LR 1997 Accounting for bias in Black-Scholes Working paper New York University

[5] Bakshi G, Cao C and Chen ZW 1997 Empirical performance of alternative option pricing models J. Finance 52 2003-49

[6] Bakshi G and Madan D 2000 Spanning and derivative-security valuation J. Financial Economics 55 205-38

[7] Black F and Scholes M 1973 The pricing of options and corporate liabilities J. Political Economy 81 637-54

[8] Bollen N PB and Whaley R E 2004 Does net buying pressure affect the shape of implied volatility functions? J. Finance 59 711-53

[9] Bouchaud JP and Potters M 2003 Theory of Financial Risk and Derivative Pricing: From Statistical Physics to Risk Management 2nd ed. (Cambridge: Cambridge University Press)

[10] Breeden D T and Litzenberger R H 1978 Prices of state-contingent claims implicit in option prices J. Business 51 621-51

[11] Brenner M, Ou E Y and Zhang J E 2006 Hedging volatility risk J. Banking Finance $30811-21$

[12] Carr P, Geman H, Madan D B and Yor M 2003 Stochastic volatility for Lévy processes Math. Finance 13 345-82

[13] Carr P and Wu LR 2003 The finite moment log stable process and option pricing $J$. Finance 58 753-77

[14] Carr P and Wu LR 2004 Time-changed Lévy processes and option pricing J. Financial Economics 17 113-41

[15] Carr P and Wu LR 2006 A tale of two indices J. Derivatives 13 13-29 
[16] Cont R and da Fonseca J 2002 Dynamics of implied volatility surfaces Quant. Finance $245-60$

[17] Corrado C J and Su T 1997 Implied volatility skews and stock index skewness and kurtosis in S\&P 500 index option prices J. Derivatives 4 8-19

[18] Cox J 1975 Notes on option pricing I: Constant elasticity of variance diffusions Working paper Stanford University

[19] Cox J and Ross S A 1976 The valuation of options for alternative stochastic processes J. Financial Economics 3 145-66

[20] Das S R and Sundaram R K 1999 Of smiles and smirks: A term structure perspective J. Financial Quant. Anal. 34 211-39

[21] Dennis P and Mayhew S 2002 Risk-neutral skewness: Evidence from stock options $J$. Financial Quant. Anal. 37 471-93

[22] Derman E and Kani I 1994 Riding on a smile Risk 7 February 32-9

[23] Duffie D, Pan J and Singleton K 2000 Transformation analysis and asset pricing for affine jump diffusions Econometrica 68 1343-76

[24] Dumas B, Fleming J and Whaley R E 1998 Implied volatility functions: Empirical tests J. Finance 53 2059-2106.

[25] Dupire B 1994 Pricing with a smile Risk 7 January 18-20

[26] Emanuel D C and MacBeth J D 1982 Further results on the constant elasticity of variance call option pricing model J. Financial Quant. Anal. 17 533-54

[27] Eraker B 2004 Do stock prices and volatility jump? Reconciling evidence from spot and option prices J. Finance 59 1367-403

[28] Feller W 1951 Two singular diffusion problems Annals of Mathematics 54 173-82 
[29] Foresi S and Wu LR 2005 Crash-o-phobia: A domestic fear or a worldwide concern J. Derivatives 13 8-21

[30] Hagan P S, Kumar D, Lesniewski A S and Woodward D E 2002 Managing smile risk Wilmott Magazine September 84-108

[31] Harrison, J M and Kreps D M 1979 Matingales and arbitrage in multiperiod securities markets J. Economic Theory 20 381-408.

[32] Harrison, J M and Pliska S R 1981 Martingales and stochastic integrals in the theory of continuous trading Stochastic Processes and Their Applications 11 215-60

[33] Heston S 1993 Closed-form solution for options with stochastic volatility, with application to bond and currency options Rev. Financial Studies 6 327-43

[34] Heynen R, Kemna A and Vorst T 1994 Analysis of the term structure of implied volatilities J. Financial Quant. Anal. 29 31-56

[35] Huang JZ and Wu LR 2004 Specification analysis of option pricing models based on time-changed Lévy processes J. Finance 59 1405-39

[36] Jackwerth J C and Rubinstein M 1996 Recovering probability distributions from option prices J. Finance 51 1611-31

[37] Jarrow R and Rudd A 1982 Approximate option valuation for arbitrary stochastic processes J. Financial Economics 10 347-69

[38] Jiang LS, Chen QH, Wang LJ and Zhang J E 2003 A new well-posed algorithm to recover implied local volatility Quant. Finance 3 451-7.

[39] Johnson N L and Kotz S 1970, Distributions in Statistics: Continuous Univariate Distribution - 2. (New York: Wiley)

[40] Kolassa J E 1997 Series Approximation Methods in Statistics 2nd ed. (New York: Springer-Verlag) 
[41] Madan D, Carr P and Chang E 1998 The variance Gamma process and option pricing European Finance Rev. 2 79-105.

[42] Merton R C 1976 Option pricing when underlying stock returns are discontinuous $J$. Financial Economics 3 125-44

[43] Navatte P and Villa C 2000 The information content of implied volatility, skewness and kurtosis: empirical evidence from long-term CAC 40 options European Financial Management 6 41-56

[44] Ncube M 1996 Modelling implied volatility with OLS and panel data models J Banking Finance 20 71-84

[45] Peña I, Rubio G and Serna G 1999 Why do we smile? On the determinants of the implied volatility function J. Banking Finance 23 1151-79

[46] Potters M, Cont R and Bouchaud JP 1998 Financial markets as adaptive systems Europhys. Lett. 41 239-44.

[47] Rubinstein M 1985 Nonparametric tests of alternative option pricing models using all reported trades and quotes on the 30 most active CBOE option classes from August 23, 1976 through August 31, 1978 J. Finance 40 455-80

[48] Rubinstein M 1994 Implied binomial trees J. Finance 49 771-818

[49] Schroder M 1989 Computing the constant elasticity of variance option pricing formula J. Finance 44 211-19

[50] Shimko D 1993 Bounds probability Risk 6 33-7.

[51] Skiadopoulos G, Hodges S and Clewlow L 1999 The dynamics of the S\&P 500 implied volatility surface Review Derivatives Research 3 263-82

[52] Xu XZ and Taylor S J 1994 The term structure of volatility implied by foreigh exchange options J. Financial Quant. Anal. 29 57-74 
[53] Zhang J E and Zhu YZ 2006 VIX futures J. Futures Markets 26 521-31

[54] Zhu YZ and Avellaneda M 1997 An E-ARCH model for the term structure of implied volatility of FX options Appl. Math. Finance 4 81-100

[55] Zhu YZ and Zhang J E 2007 Variance term structure and VIX futures pricing Int. J. Theor. Appl. Finance 10 1-18 
Table 1: The market data of SPX options on November 4, 2003. The maturity date is November 21, 2003. The time to maturity is $\tau=17$ days, or $17 / 365$ year. The S\&P 500 index level is $S_{0}=1053.25$. The risk-free rate is $r=0.9743 \%$. The implied forward index level, computed by using formula $F_{0}=K+e^{r \tau}\left(c_{0}(K)-p_{0}(K)\right)$ with $K=1055$ to minimize $\left|c_{0}(K)-p_{0}(K)\right|$, is $F_{0}=1052.70$. The fact of $F_{0}<S_{0}$ implies that the expected dividend yield, $q=r-\ln \left(F_{0} / S_{0}\right)$, is larger than the risk-free rate, $r$.

\begin{tabular}{|c|c|c|c|c|c|c|c|c|c|c|}
\hline \multirow[b]{2}{*}{ Strike } & \multicolumn{5}{|c|}{ Call } & \multicolumn{5}{|c|}{ Put } \\
\hline & Last Sale & Bid & Ask & Mid-value & Vol & Last Sale & Bid & Ask & Mid-value & Vol \\
\hline 850 & 204.6 & 201.6 & 203.6 & 202.6 & 0 & 0.1 & 0.05 & 0.15 & 0.1 & 0 \\
\hline 875 & 160.4 & 176.7 & 178.7 & 177.7 & 0 & 0.15 & 0.1 & 0.3 & 0.2 & 0 \\
\hline 900 & 150 & 151.8 & 153.8 & 152.8 & 0 & 0.2 & 0.15 & 0.25 & 0.2 & 0 \\
\hline 925 & 135 & 126.9 & 128.9 & 127.9 & 0 & 0.3 & 0.3 & 0.5 & 0.4 & 307 \\
\hline 935 & 124 & 116.9 & 118.9 & 117.9 & 0 & 0.55 & 0.25 & 0.55 & 0.4 & 26 \\
\hline 945 & 0 & 107 & 109 & 108 & 0 & 1.3 & 0.35 & 0.65 & 0.5 & 0 \\
\hline 950 & 102.5 & 102.1 & 104.1 & 103.1 & 10 & 0.55 & 0.45 & 0.6 & 0.525 & 832 \\
\hline 960 & 0 & 92.2 & 94.2 & 93.2 & 0 & 0.65 & 0.35 & 0.8 & 0.575 & 0 \\
\hline 970 & 0 & 82.4 & 84.4 & 83.4 & 0 & 0 & 0.5 & 1 & 0.75 & 0 \\
\hline 975 & 82 & 77.6 & 79.6 & 78.6 & 0 & 1 & 0.8 & 1.1 & 0.95 & 1362 \\
\hline 980 & 72.5 & 72.7 & 74.7 & 73.7 & 1 & 1.05 & 0.8 & 1.3 & 1.05 & 34 \\
\hline 985 & 63 & 67.9 & 69.9 & 68.9 & 0 & 1.3 & 1 & 1.45 & 1.225 & 347 \\
\hline 990 & 70.5 & 63.1 & 65.1 & 64.1 & 0 & 1.4 & 1.2 & 1.65 & 1.425 & 188 \\
\hline 995 & 58.6 & 58.3 & 60.3 & 59.3 & 13 & 1.7 & 1.6 & 1.9 & 1.75 & 1109 \\
\hline 1005 & 49 & 49 & 51 & 50 & 45 & 2.3 & 2.25 & 2.6 & 2.425 & 493 \\
\hline 1010 & 44.6 & 44.5 & 46.5 & 45.5 & 146 & 2.9 & 2.4 & 3.2 & 2.8 & 190 \\
\hline 1015 & 40 & 40 & 42 & 41 & 29 & 3.7 & 2.9 & 3.7 & 3.3 & 965 \\
\hline 1020 & 39.7 & 35.7 & 37.7 & 36.7 & 6 & 3.6 & 3.6 & 4.3 & 3.95 & 1118 \\
\hline 1025 & 31.9 & 31.5 & 33.5 & 32.5 & 1952 & 4.6 & 4.5 & 5.1 & 4.8 & 2005 \\
\hline 1030 & 28.5 & 27.4 & 29.4 & 28.4 & 9 & 5.5 & 5.1 & 6 & 5.55 & 373 \\
\hline 1035 & 23.7 & 24 & 25.6 & 24.8 & 187 & 7.2 & 6.3 & 7.5 & 6.9 & 2525 \\
\hline 1040 & 20.3 & 20 & 22 & 21 & 107 & 8.2 & 7.7 & 8.5 & 8.1 & 1190 \\
\hline 1045 & 17 & 17 & 18.5 & 17.75 & 14 & 10.1 & 9.4 & 10.6 & 10 & 94 \\
\hline 1050 & 14.5 & 14.5 & 15.4 & 14.95 & 3033 & 11.9 & 11.6 & 12.2 & 11.9 & 3478 \\
\hline 1055 & 11.5 & 11.1 & 12.7 & 11.9 & 1823 & 14.5 & 13.4 & 15 & 14.2 & 627 \\
\hline 1060 & 9.8 & 9.5 & 10 & 9.75 & 2603 & 17 & 16 & 17.2 & 16.6 & 188 \\
\hline 1065 & 7.1 & 7.2 & 8.1 & 7.65 & 243 & 20 & 19 & 20.6 & 19.8 & 24 \\
\hline 1070 & 5.5 & 5.4 & 6.4 & 5.9 & 2623 & 23.7 & 22.1 & 24.1 & 23.1 & 204 \\
\hline 1075 & 4.1 & 4.1 & 4.7 & 4.4 & 1759 & 26.8 & 25.7 & 27.2 & 26.45 & 1704 \\
\hline 1080 & 3.2 & 3.1 & 3.7 & 3.4 & 303 & 30 & 29.6 & 31.6 & 30.6 & 6 \\
\hline 1085 & 2.4 & 2 & 2.8 & 2.4 & 79 & 0 & 33.7 & 35.7 & 34.7 & 0 \\
\hline 1090 & 1.9 & 1.5 & 2 & 1.75 & 362 & 40 & 38 & 40 & 39 & 2 \\
\hline 1095 & 1.1 & 1 & 1.5 & 1.25 & 10 & 0 & 42.5 & 44.5 & 43.5 & 0 \\
\hline 1100 & 0.95 & 0.85 & 1.1 & 0.975 & 149 & 49 & 47.2 & 49.2 & 48.2 & 42 \\
\hline 1115 & 0.45 & 0.3 & 0.5 & 0.4 & 32 & 0 & 61.6 & 63.6 & 62.6 & 0 \\
\hline 1125 & 0.25 & 0.15 & 0.3 & 0.225 & 39 & 72 & 71.4 & 73.4 & 72.4 & 13 \\
\hline
\end{tabular}


Table 2: The U.S. daily treasury yield curve rates (\%) between October 30, 2003 and November 12, 2003.

\begin{tabular}{|l|cccccccccc|}
\hline Mate & $1 \mathrm{mo}$ & $3 \mathrm{mo}$ & $6 \mathrm{mo}$ & $1 \mathrm{yr}$ & $2 \mathrm{yr}$ & $3 \mathrm{yr}$ & $5 \mathrm{yr}$ & $7 \mathrm{yr}$ & $10 \mathrm{yr}$ & $20 \mathrm{yr}$ \\
\hline $10 / 30 / 03$ & 0.97 & 0.96 & 1.04 & 1.32 & 1.86 & 2.39 & 3.29 & 3.83 & 4.36 & 5.25 \\
$10 / 31 / 03$ & 0.96 & 0.96 & 1.04 & 1.31 & 1.85 & 2.36 & 3.27 & 3.80 & 4.33 & 5.20 \\
$11 / 03 / 03$ & 0.97 & 0.96 & 1.05 & 1.33 & 1.90 & 2.44 & 3.34 & 3.88 & 4.40 & 5.25 \\
$11 / 04 / 03$ & 0.97 & 0.95 & 1.03 & 1.31 & 1.86 & 2.40 & 3.28 & 3.81 & 4.33 & 5.19 \\
$11 / 05 / 03$ & 0.96 & 0.96 & 1.05 & 1.35 & 1.94 & 2.46 & 3.35 & 3.88 & 4.38 & 5.24 \\
$11 / 06 / 03$ & 0.95 & 0.96 & 1.06 & 1.38 & 2.01 & 2.55 & 3.43 & 3.96 & 4.45 & 5.32 \\
$11 / 07 / 03$ & 0.95 & 0.96 & 1.07 & 1.40 & 2.04 & 2.60 & 3.47 & 3.99 & 4.48 & 5.33 \\
$11 / 10 / 03$ & 0.92 & 0.97 & 1.07 & 1.39 & 2.06 & 2.63 & 3.49 & 4.00 & 4.49 & 5.34 \\
$11 / 12 / 03$ & 0.92 & 0.95 & 1.06 & 1.39 & 2.05 & 2.59 & 3.45 & 3.95 & 4.44 & 5.29 \\
\hline
\end{tabular}


Table 3: The price and implied volatility on November 4, 2003 for out-of-the-money SPX options that mature on November 21, 2003. Puts are chosen for strikes below the forward index level, $F_{0}=1052.70$. Calls are chosen for strike above the forward index level. The implied volatility is the root that equates the Black-Scholes formula with the market price, and the moneyness is defined as $\ln \left(K / F_{0}\right) /(\bar{\sigma} \sqrt{\tau})$, where $\tau=17 / 365$ year, and $\bar{\sigma}=16.55 \%$ is the VIX closing index on the day. It is taken to be a proxy of the average volatility. The fitted implied volatility, given by $I V=0.1447\left(1-0.1308 \xi+0.0411 \xi^{2}\right)$, is obtained by fitting the implied volatility with a quadratic function that passes through the point atthe-money and minimizes the volume weighted mean squared error. RMSE, root of mean squared error. RVWMSE, root of volume weighted mean squared error.

\begin{tabular}{|c|c|c|c|c|c|c|c|}
\hline & Strike & Market & Volume & Moneyness & IV. & Fitted IV. & FitEr \\
\hline \multirow{24}{*}{ Put } & 850 & 0.1 & 0 & -5.9881 & 0.3760 & 0.4713 & 0.0953 \\
\hline & 875 & 0.2 & 0 & -5.1765 & 0.3568 & 0.4020 & 0.0452 \\
\hline & 900 & 0.2 & 0 & -4.3878 & 0.3081 & 0.3422 & 0.0341 \\
\hline & 925 & 0.4 & 307 & -3.6206 & 0.2861 & 0.2911 & 0.0050 \\
\hline & 935 & 0.4 & 26 & -3.3196 & 0.2655 & 0.2730 & 0.0075 \\
\hline & 945 & 0.5 & 0 & -3.0217 & 0.2537 & 0.2561 & 0.0025 \\
\hline & 950 & 0.525 & 832 & -2.8740 & 0.2451 & 0.2482 & 0.0031 \\
\hline & 960 & 0.575 & 0 & -2.5808 & 0.2273 & 0.2331 & 0.0058 \\
\hline & 970 & 0.75 & 0 & -2.2907 & 0.2159 & 0.2192 & 0.0033 \\
\hline & 975 & 0.95 & 1362 & -2.1467 & 0.2144 & 0.2127 & -0.0022 \\
\hline & 980 & 1.05 & 34 & -2.0035 & 0.2070 & 0.2064 & -0.0006 \\
\hline & 985 & 1.225 & 347 & -1.8610 & 0.2019 & 0.2005 & -0.0014 \\
\hline & 990 & 1.425 & 188 & -1.7193 & 0.1965 & 0.1948 & -0.0017 \\
\hline & 995 & 1.75 & 1109 & -1.5782 & 0.1936 & 0.1893 & -0.0043 \\
\hline & 1005 & 2.425 & 493 & -1.2983 & 0.1835 & 0.1792 & -0.0043 \\
\hline & 1010 & 2.8 & 190 & -1.1593 & 0.1773 & 0.1746 & -0.0027 \\
\hline & 1015 & 3.3 & 965 & -1.0210 & 0.1719 & 0.1702 & -0.0018 \\
\hline & 1020 & 3.95 & 1118 & -0.8835 & 0.1675 & 0.1660 & -0.0015 \\
\hline & 1025 & 4.8 & 2005 & -0.7466 & 0.1641 & 0.1621 & -0.0020 \\
\hline & 1030 & 5.55 & 373 & -0.6103 & 0.1571 & 0.1584 & 0.0013 \\
\hline & 1035 & 6.9 & 2525 & -0.4747 & 0.1559 & 0.1550 & -0.0009 \\
\hline & 1040 & 8.1 & 1190 & -0.3398 & 0.150 & 0.1518 & 0.0017 \\
\hline & 1045 & 10 & 94 & -0.2055 & 0.1498 & 0.1488 & -0.0008 \\
\hline & 1050 & 11.9 & 3478 & -0.0719 & 0.1460 & 0.1460 & 0.0001 \\
\hline ATM & 1052.70 & & & -0.0001 & 0.1447 & 0.1447 & 0.0000 \\
\hline \multirow{12}{*}{ Call } & 1055 & 11.9 & 1823 & 0.0611 & 0.1435 & 0.1435 & -0.00003 \\
\hline & 1060 & 9.75 & 2603 & 0.1935 & 0.1439 & 0.1412 & -0.0026 \\
\hline & 1065 & 7.65 & 243 & 0.3253 & 0.1413 & 0.1391 & -0.0022 \\
\hline & 1070 & 5.9 & 2623 & 0.4564 & 0.1393 & 0.1373 & -0.0020 \\
\hline & 1075 & 4.4 & 1759 & 0.5869 & 0.1367 & 0.1356 & -0.0011 \\
\hline & 1080 & 3.4 & 303 & 0.7169 & 0.1375 & 0.1342 & -0.0034 \\
\hline & 1085 & 2.4 & 79 & 0.8462 & 0.1348 & 0.1329 & -0.0018 \\
\hline & 1090 & 1.75 & 362 & 0.9749 & 0.1346 & 0.1319 & -0.0028 \\
\hline & 1095 & 1.25 & 10 & 1.1030 & 0.1344 & 0.1310 & -0.0034 \\
\hline & 1100 & 0.975 & 149 & 1.2306 & 0.1375 & 0.1304 & -0.0071 \\
\hline & 1115 & 0.4 & 32 & 1.6098 & 0.1426 & 0.1296 & -0.0130 \\
\hline & 1125 & 0.225 & 39 & 1.8598 & 0.1468 & 0.1301 & -0.0168 \\
\hline RMSE & & & & & & & 0.0190 \\
\hline RVWMSE & & & & & & & 0.0023 \\
\hline
\end{tabular}


Table 4: The prices and price errors computed by the Black-Scholes formula with flat, skewed and smirked volatility functions for out-of-the-money SPX options. The set of options used is the same as that in Table 3. The flat volatility function, $I V=0.1447$, is a constant given by at-the-money implied volatility. The skewed one, $I V=0.1447(1-$ $0.1308 \xi)$, is a linear function passing through the point at the money. The smirked one, $I V=0.1447\left(1-0.1308 \xi+0.0411 \xi^{2}\right)$, is a quadratic function obtained by fitting the implied volatility. The errors are the difference between the computed prices and the market prices. RMSE, root of mean squared error. RVWMSE, root of volume weighted mean squared error.

\begin{tabular}{|c|c|c|c|c|c|c|c|c|}
\hline Strike & Market & Volume & Flat & FlatEr & Skew & SkewEr & Smirk & SmirkEr \\
\hline 850 & 0.1 & 0 & 0 & -0.1 & 0.0008 & -0.0993 & 0.6163 & 0.5163 \\
\hline 875 & 0.2 & 0 & 0 & -0.2 & 0.0026 & -0.1974 & 0.4933 & 0.2933 \\
\hline 900 & 0.2 & 0 & 0 & -0.2 & 0.0092 & -0.1908 & 0.4368 & 0.2368 \\
\hline 925 & 0.4 & 307 & 0.0001 & -0.3999 & 0.0332 & -0.3668 & 0.4493 & 0.0493 \\
\hline 935 & 0.4 & 26 & 0.0005 & -0.3995 & 0.0556 & -0.3444 & 0.4797 & 0.0797 \\
\hline 945 & 0.5 & 0 & 0.0022 & -0.4979 & 0.0931 & -0.4069 & 0.5307 & 0.0307 \\
\hline 950 & 0.525 & 832 & 0.0042 & -0.5209 & 0.1203 & -0.4047 & 0.5664 & 0.0414 \\
\hline 960 & 0.575 & 0 & 0.0142 & -0.5609 & 0.2005 & -0.3745 & 0.6646 & 0.0896 \\
\hline 970 & 0.75 & 0 & 0.0432 & -0.7068 & 0.3325 & -0.4175 & 0.8127 & 0.0627 \\
\hline 975 & 0.95 & 1362 & 0.0725 & -0.8775 & 0.4272 & -0.5228 & 0.9127 & -0.0373 \\
\hline 980 & 1.05 & 34 & 0.1187 & -0.9313 & 0.5477 & -0.5023 & 1.0355 & -0.0145 \\
\hline 985 & 1.225 & 347 & 0.1895 & -1.0355 & 0.7006 & -0.5244 & 1.1866 & -0.0384 \\
\hline 990 & 1.425 & 188 & 0.2953 & -1.1298 & 0.8939 & -0.5312 & 1.3727 & -0.0523 \\
\hline 995 & 1.75 & 1109 & 0.4494 & -1.3007 & 1.1371 & -0.6129 & 1.6024 & -0.1476 \\
\hline 1005 & 2.425 & 493 & 0.9723 & -1.4527 & 1.8217 & -0.6033 & 2.2365 & -0.1886 \\
\hline 1010 & 2.8 & 190 & 1.3844 & -1.4156 & 2.2925 & -0.5075 & 2.6693 & -0.1307 \\
\hline 1015 & 3.3 & 965 & 1.9306 & -1.3694 & 2.8723 & -0.4277 & 3.2030 & -0.0970 \\
\hline 1020 & 3.95 & 1118 & 2.6388 & -1.3112 & 3.5816 & -0.3684 & 3.8595 & -0.0906 \\
\hline 1025 & 4.8 & 2005 & 3.5377 & -1.2623 & 4.4431 & -0.3569 & 4.6634 & -0.1366 \\
\hline 1030 & 5.55 & 373 & 4.6557 & -0.8943 & 5.4810 & -0.0690 & 5.6425 & 0.0925 \\
\hline 1035 & 6.9 & 2525 & 6.0190 & -0.8811 & 6.7208 & -0.1792 & 6.8266 & -0.0734 \\
\hline 1040 & 8.1 & 1190 & 7.6503 & -0.4497 & 8.1885 & 0.0885 & 8.2462 & 0.1462 \\
\hline 1045 & 10 & 94 & 9.5675 & -0.4325 & 9.9090 & -0.0910 & 9.9311 & -0.0689 \\
\hline 1050 & 11.9 & 3478 & 11.7824 & -0.1178 & 11.9050 & 0.0050 & 11.9078 & 0.0078 \\
\hline 1052.70 & & & & 0.0000 & & 0.0000 & & 0.0000 \\
\hline 1055 & 11.9 & 1823 & 12.0003 & 0.1103 & 11.8957 & -0.0043 & 11.8977 & -0.0023 \\
\hline 1060 & 9.75 & 2603 & 9.8214 & 0.0714 & 9.4970 & -0.2530 & 9.5167 & -0.2333 \\
\hline 1065 & 7.65 & 243 & 7.9350 & 0.2849 & 7.4135 & -0.2365 & 7.4667 & -0.1833 \\
\hline 1070 & 5.9 & 2623 & 6.3262 & 0.4262 & 5.6442 & -0.2558 & 5.7414 & -0.1586 \\
\hline 1075 & 4.4 & 1759 & 4.9754 & 0.5754 & 4.1794 & -0.2207 & 4.3239 & -0.0761 \\
\hline 1080 & 3.4 & 303 & 3.8590 & 0.4590 & 3.0005 & -0.3995 & 3.1885 & -0.2115 \\
\hline 1085 & 2.4 & 79 & 2.9510 & 0.5510 & 2.0815 & -0.3186 & 2.3023 & -0.0978 \\
\hline 1090 & 1.75 & 362 & 2.2244 & 0.4744 & 1.3900 & -0.3600 & 1.6285 & -0.1215 \\
\hline 1095 & 1.25 & 10 & 1.6525 & 0.4025 & 0.8898 & -0.3602 & 1.1294 & -0.1206 \\
\hline 1100 & 0.975 & 149 & 1.2097 & 0.2347 & 0.5436 & -0.4314 & 0.7689 & -0.2061 \\
\hline 1115 & 0.4 & 32 & 0.4341 & 0.0341 & 0.0887 & -0.3113 & 0.2215 & -0.1785 \\
\hline 1125 & 0.225 & 39 & 0.2033 & -0.0217 & 0.0188 & -0.2062 & 0.0918 & -0.1332 \\
\hline RMSE & & & & 0.7504 & & 0.3591 & & 0.1566 \\
\hline RVWMSE & & & & 0.7758 & & 0.3127 & & 0.1229 \\
\hline
\end{tabular}


Table 5: The term structure of smirk parameters on November 4, 2003. The trading volume is the number of the out-of-the-money options with different strikes for the same maturity. The discount rate is computed by using linear interpolation with the yield curve on November 4, 2003 provided in Table 2. The smirk parameter set $\left(\gamma_{0}, \gamma_{1}, \gamma_{2}\right)$ is obtained by fitting the implied volatility smirk with a quadratic function, $I V(\xi)=\gamma_{0}\left(1+\gamma_{1} \xi+\gamma_{2} \xi^{2}\right)$, where $\xi$ is the moneyness.

\begin{tabular}{|c|cc|ccc|}
\hline $\begin{array}{c}\text { Maturity } \\
\tau \text { (days) }\end{array}$ & $\begin{array}{c}\text { Trading } \\
\text { volume }\end{array}$ & $\begin{array}{c}\text { Discount rate } \\
r(\%)\end{array}$ & $\begin{array}{c}\text { ATMV } \\
\gamma_{0}\end{array}$ & $\begin{array}{c}\text { Slope } \\
\gamma_{1}\end{array}$ & $\begin{array}{c}\text { Curvature } \\
\gamma_{2}\end{array}$ \\
\hline 17 & 26,661 & 0.9743 & 0.1447 & -0.1308 & 0.0411 \\
45 & 37,983 & 0.9651 & 0.1473 & -0.1894 & 0.0158 \\
73 & 4,066 & 0.9559 & 0.1509 & -0.2141 & 0.0103 \\
136 & 6,825 & 0.9896 & 0.1608 & -0.2063 & 0.0049 \\
227 & 4,848 & 1.0989 & 0.1683 & -0.1623 & 0.0230 \\
318 & 4,050 & 1.2381 & 0.1727 & -0.1905 & 0.0425 \\
409 & 3,987 & 1.3763 & 0.1759 & -0.1688 & 0.0086 \\
591 & 1,401 & 1.6506 & 0.1786 & -0.1574 & 0.0309 \\
\hline
\end{tabular}


Table 6: The evolution of the term structure of smirk parameters from October 30, 2003 to November 4, 2003 and then to November 12, 2003. The discount rate is computed by using linear interpolation with the yield curve on the corresponding dates provided in Table 2 . The smirk parameter set $\left(\gamma_{0}, \gamma_{1}, \gamma_{2}\right)$ is obtained by fitting the implied volatility smirk with a quadratic function, $I V(\xi)=\gamma_{0}\left(1+\gamma_{1} \xi+\gamma_{2} \xi^{2}\right)$, where $\xi$ is the moneyness.

Observed on October 30, 2003

\begin{tabular}{|c|cccccccc|}
\hline Maturity (days) & 22 & 50 & 78 & 141 & 232 & 323 & 414 & 596 \\
\hline Discount rate & 0.9713 & 0.9667 & 0.9621 & 1.0040 & 1.1165 & 1.2557 & 1.3925 & 1.6618 \\
\hline ATMV $\gamma_{0}$ & 0.1474 & 0.1528 & 0.1568 & 0.1661 & 0.1729 & 0.1776 & 0.1805 & 0.1833 \\
\hline Slope $\gamma_{1}$ & -0.1599 & -0.1820 & -0.1648 & -0.1606 & -0.1765 & -0.1856 & -0.1988 & -0.2096 \\
\hline Curvature $\gamma_{2}$ & 0.0220 & 0.0103 & 0.0190 & 0.0250 & 0.0109 & 0.0108 & -0.0055 & -0.0103 \\
\hline
\end{tabular}

Observed on November 4, 2003

\begin{tabular}{|c|cccccccc|}
\hline Maturity (days) & 17 & 45 & 73 & 136 & 227 & 318 & 409 & 591 \\
\hline Discount rate & 0.9743 & 0.9651 & 0.9559 & 0.9896 & 1.0989 & 1.2381 & 1.3763 & 1.6506 \\
\hline ATMV $\gamma_{0}$ & 0.1447 & 0.1473 & 0.1509 & 0.1608 & 0.1683 & 0.1727 & 0.1759 & 0.1786 \\
\hline Slope $\gamma_{1}$ & -0.1308 & -0.1894 & -0.2141 & -0.2063 & -0.1623 & -0.1905 & -0.1688 & -0.1574 \\
\hline Curvature $\gamma_{2}$ & 0.0411 & 0.0158 & 0.0103 & 0.0049 & 0.0230 & 0.0425 & 0.0086 & 0.0309 \\
\hline
\end{tabular}

Observed on November 12, 2003

\begin{tabular}{|c|cccccccc|}
\hline Maturity (days) & 9 & 37 & 65 & 128 & 219 & 310 & 401 & 583 \\
\hline Discount rate & 0.9097 & 0.9234 & 0.9372 & 0.9947 & 1.1267 & 1.2908 & 1.4551 & 1.7842 \\
\hline ATMV $\gamma_{0}$ & 0.1632 & 0.1553 & 0.1575 & 0.1658 & 0.1712 & 0.1743 & 0.1776 & 0.1798 \\
\hline Slope $\gamma_{1}$ & -0.1357 & -0.1723 & -0.1719 & -0.2062 & -0.2117 & -0.2052 & -0.1867 & -0.1838 \\
\hline Curvature $\gamma_{2}$ & 0.0256 & 0.0168 & 0.0291 & 0.0063 & 0.0049 & 0.0031 & -0.0006 & 0.0188 \\
\hline
\end{tabular}


Table 7: The term structure of smirk parameters observed from the market data on November 4, 2003, and implied in calibrated option pricing models: the Constant Elasticity of Variance (CEV) model and the Finite Moment Log Stable (FMLS) process.

The term structure of smirk parameters observed from the market data on November 4, 2003

\begin{tabular}{|c|cccccccc|}
\hline Maturity (days) & 17 & 45 & 73 & 136 & 227 & 318 & 409 & 591 \\
\hline Discount rate (\%) & 0.9743 & 0.9651 & 0.9559 & 0.9896 & 1.0989 & 1.2381 & 1.3763 & 1.6506 \\
Dividend (\%) & 2.098 & 1.656 & 1.548 & 1.704 & 1.565 & 1.615 & 1.609 & 1.474 \\
Forward price & 1052.70 & 1052.35 & 1052.00 & 1050.45 & 1050.20 & 1049.80 & 1050.51 & 1056.27 \\
\hline & & & & & & & & \\
\hline ATMV $\gamma_{0}$ & 0.1447 & 0.1473 & 0.1509 & 0.1608 & 0.1683 & 0.1727 & 0.1759 & 0.1786 \\
Slope $\gamma_{1}$ & -0.1308 & -0.1894 & -0.2141 & -0.2063 & -0.1623 & -0.1905 & -0.1688 & -0.1574 \\
Curvature $\gamma_{2}$ & 0.0411 & 0.0158 & 0.0103 & 0.0049 & 0.0230 & 0.0425 & 0.0086 & 0.0309 \\
\hline
\end{tabular}

The term structure of smirk parameters implied in the CEV model, $d S_{t}=(r-q) S_{t} d t+\sigma S_{t}^{\alpha} d B_{t}$, with calibrated parameters: $(\sigma, \alpha)=(152.36,0)$

\begin{tabular}{|c|cccccccc|}
\hline Maturity (days) & 17 & 45 & 73 & 136 & 227 & 318 & 409 & 591 \\
\hline ATMV $\gamma_{0}$ & 0.1447 & 0.1447 & 0.1448 & 0.1449 & 0.1449 & 0.1450 & 0.1450 & 0.1447 \\
Slope $\gamma_{1}$ & -0.0179 & -0.0291 & -0.0370 & -0.0506 & -0.0653 & -0.0774 & -0.0878 & -0.1056 \\
Curvature $\gamma_{2}$ & 0.00011 & 0.00028 & 0.00046 & 0.00085 & 0.00143 & 0.00201 & 0.00259 & 0.00375 \\
\hline
\end{tabular}

The term structure of smirk parameters implied in the FMLS process, $d S_{t}=(r-q) S_{t} d t+\sigma S_{t} d L_{t}^{\alpha,-1}$, with calibrated parameters: $(\sigma, \alpha)=(0.1086,1.8141)$

\begin{tabular}{|c|cccccccc|}
\hline Maturity (days) & 17 & 45 & 73 & 136 & 227 & 318 & 409 & 591 \\
\hline ATMV $\gamma_{0}$ & 0.1447 & 0.1508 & 0.1537 & 0.1574 & 0.1603 & 0.1621 & 0.1634 & 0.1652 \\
Slope $\gamma_{1}$ & -0.1308 & -0.1195 & -0.1137 & -0.1059 & -0.0992 & -0.0947 & -0.0913 & -0.0863 \\
Curvature $\gamma_{2}$ & 0.0486 & 0.0416 & 0.0383 & 0.0341 & 0.0307 & 0.0285 & 0.0269 & 0.0246 \\
\hline
\end{tabular}




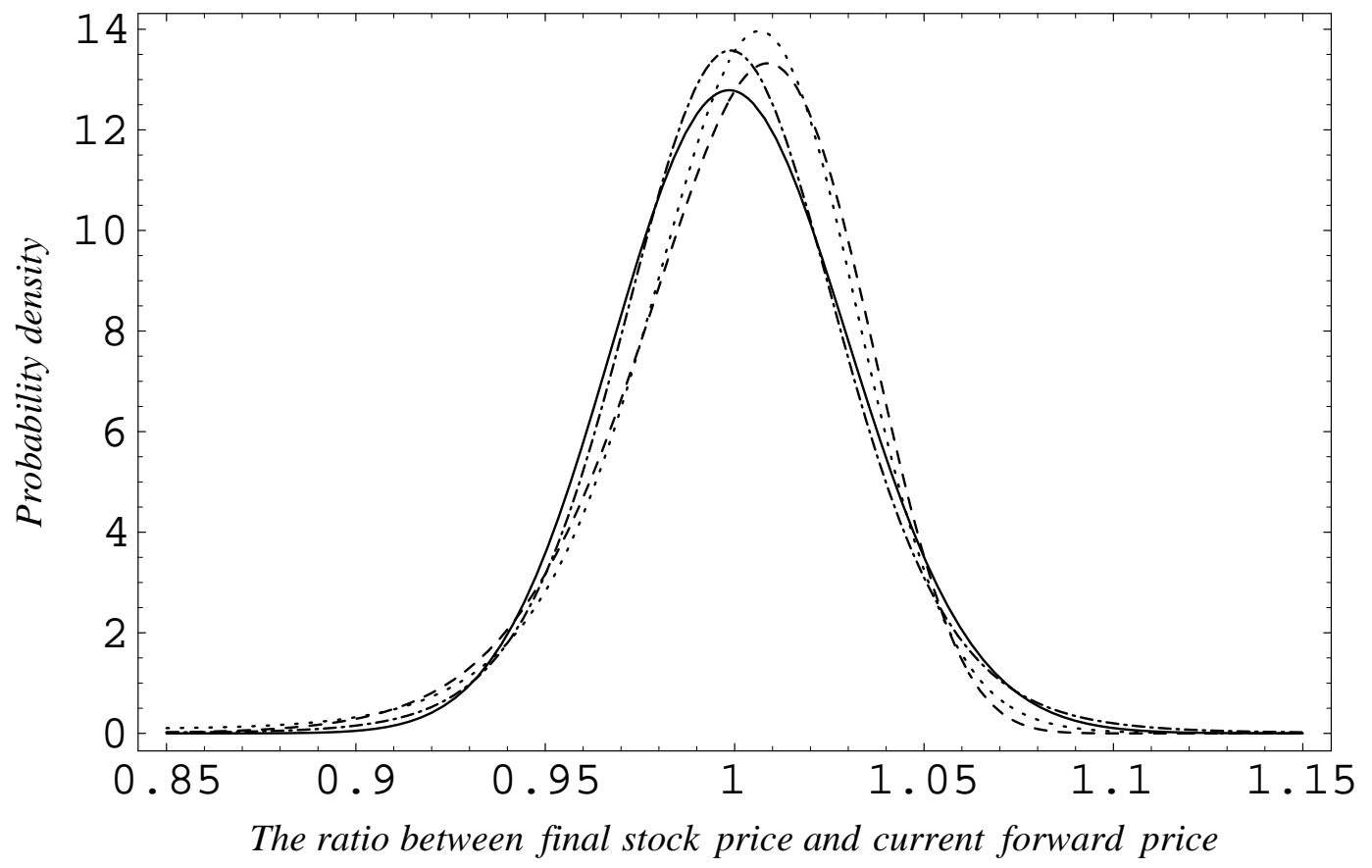

Figure 1: The risk-neutral probability density functions recovered from prices of options with 17 days to maturity and flat, skewed, smiled and smirked implied volatility functions. The solid line is the probability density function recovered from a flat implied volatility, $I V=\gamma_{0}=0.1447$. It is a lognormal distribution density function. The dashed line is recovered from a skewed implied volatility, $I V=\gamma_{0}\left(1+\gamma_{1} \xi\right)=0.1447(1-0.1308 \xi)$. The dash-doted line is recovered from a smiled implied volatility, $I V=\gamma_{0}\left(1+\gamma_{2} \xi^{2}\right)=$ $0.1447\left(1+0.0411 \xi^{2}\right)$. The doted line is recovered from a smirked implied volatility, $I V=$ $\gamma_{0}\left(1+\gamma_{1} \xi+\gamma_{2} \xi^{2}\right)=0.1447\left(1-0.1308 \xi+0.0411 \xi^{2}\right)$. The numerical values of $\gamma_{0}, \gamma_{1}$ and $\gamma_{3}$ are determined by fitting the market data on November 4, 2003 for the S\&P 500 index options that mature on November 21, 2003. 


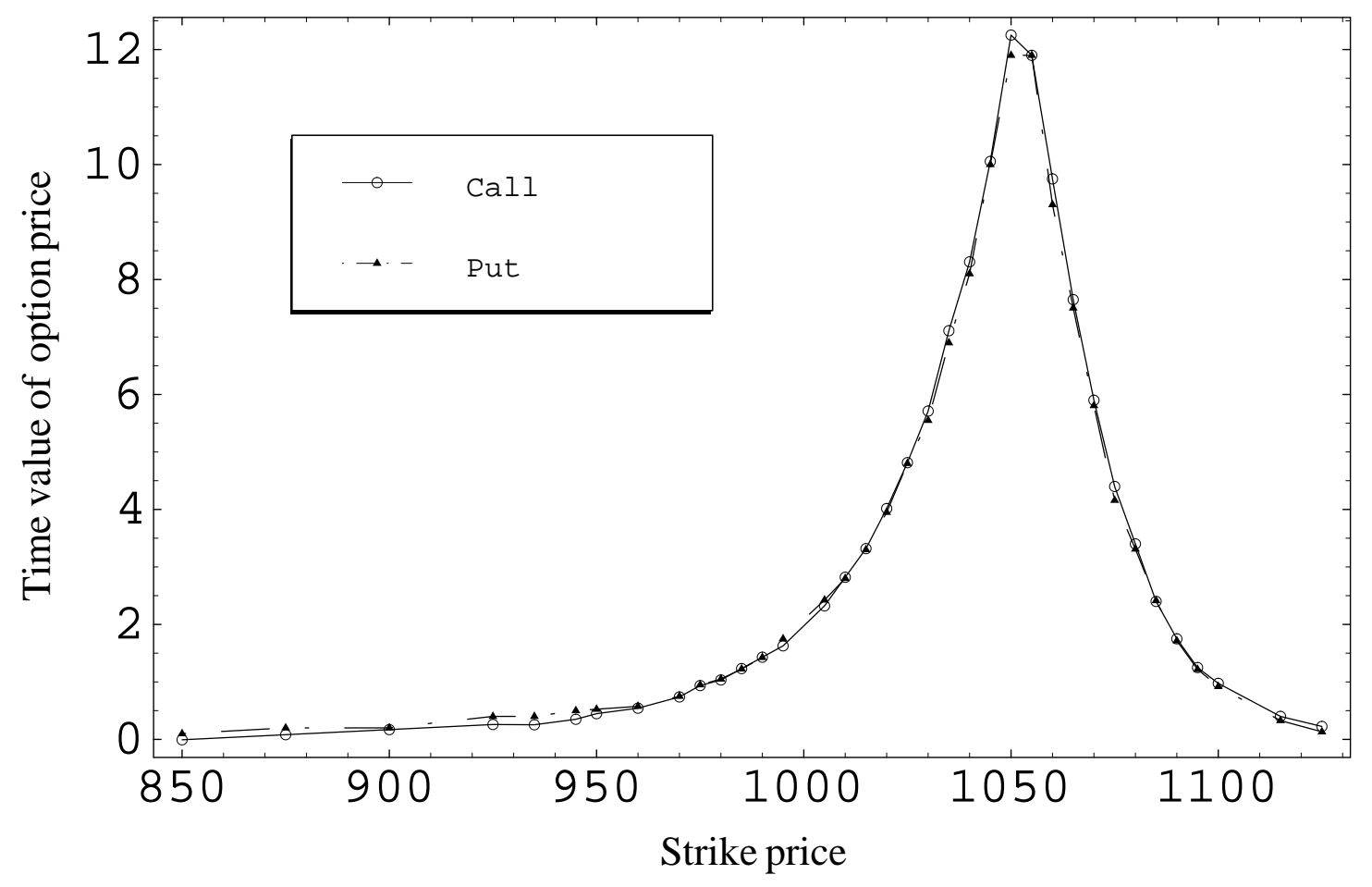

Figure 2: The time value of SPX options as a function of strike price, $K$, on November 4, 2003 for both calls and puts that mature on November 21, 2003. The time to maturity is $\tau=17$ days $=17 / 365$ year. The discount rate over the period, derived from the U.S. treasury yield curve on November 4, 2003, is $r=0.9743 \%$. The implied forward price, derived from put-call parity for the strike that minimizes the absolute difference between a call and a put price, is $F_{0}=1052.70$. The time value of an option is defined as the difference between the option price and its intrinsic value. The time value of a call is therefore $c_{t v}(K)=c_{0}(K)-e^{-r \tau} \max \left(F_{0}-K, 0\right)$, and the time value of a put is $p_{t v}(K)=p_{0}(K)-e^{-r \tau} \max \left(K-F_{0}, 0\right)$. The fact that the two functions, $c_{t v}(K)$ and $p_{t v}(K)$, almost collapse on each other indicates that the put-call parity holds for any strike price, $K$. 
Maturity 17 days

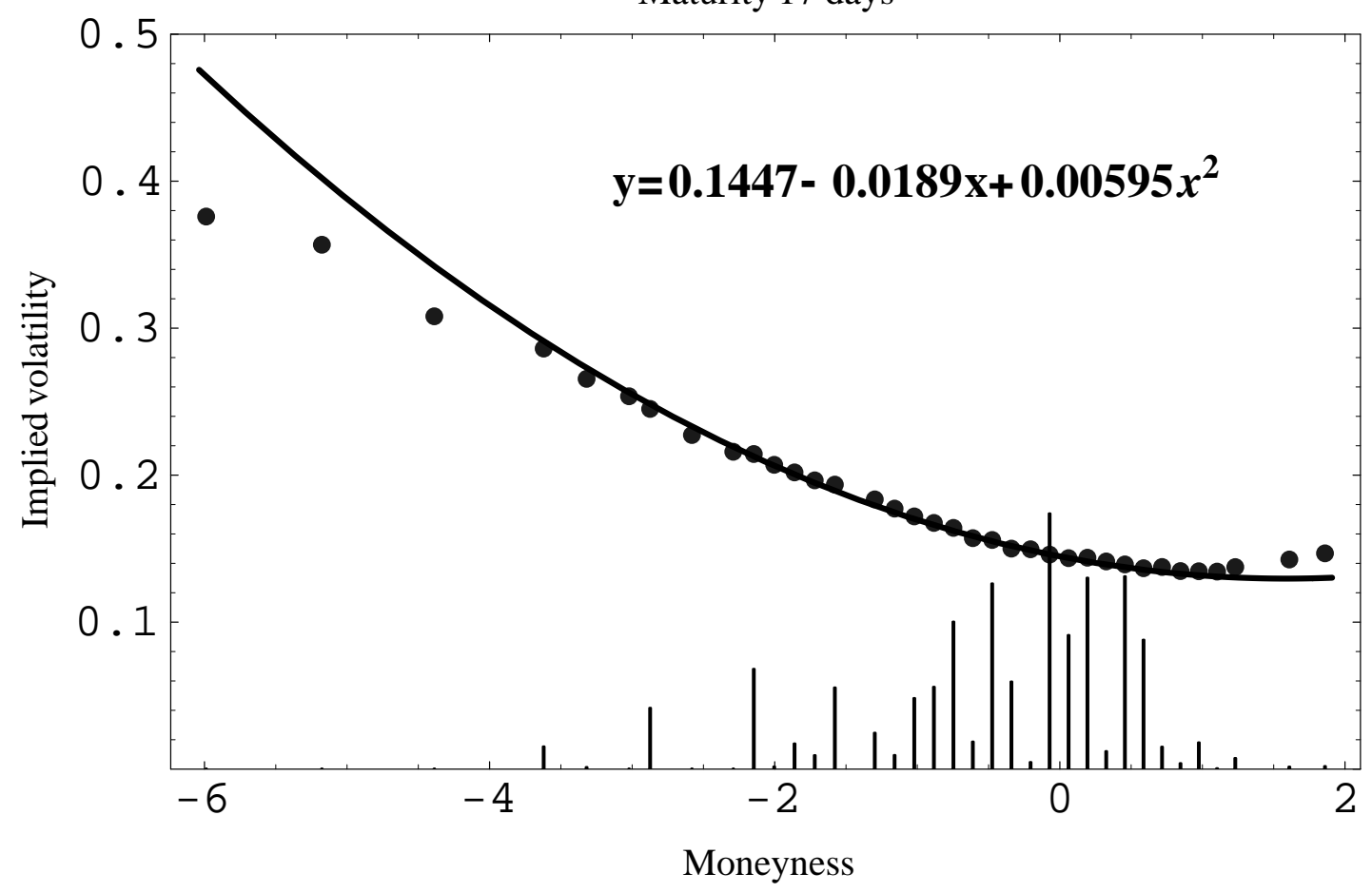

Figure 3: The implied volatility smirk on November 4, 2003 for out-of-the-money SPX options that mature on November 21, 2003. The implied volatility, $I V$, as a function of moneyness, $\xi$, is regarded as an implied volatility smirk. The implied volatility, $I V$, is defined as the root that equates the Black-Scholes formula with the market price, $F_{0} e^{-r \tau} N\left(\frac{\ln \left(F_{0} / K\right)+\frac{1}{2} I V^{2} \tau}{I V \sqrt{\tau}}\right)-K e^{-r \tau} N\left(\frac{\ln \left(F_{0} / K\right)-\frac{1}{2} I V^{2} \tau}{I V \sqrt{\tau}}\right)=c_{\mathrm{mkt}}$, and the moneyness, $\xi$, is defined by $\xi \equiv \frac{\ln \left(K / F_{0}\right)}{\bar{\sigma} \sqrt{\tau}}$. Here, the time to maturity is $\tau=17$ days $=17 / 365$ year. The discount rate over the period, derived from the U.S. treasury yield curve on November 4, 2003, is $r=0.9743 \%$. The implied forward price, derived from put-call parity for the strike that minimizes the absolute difference between a call and a put prices, is $F_{0}=1052.70$. The benchmark volatility, $\bar{\sigma}$, is taken to be the VIX index reported by the CBOE on the day, $\bar{\sigma}=16.55 \%$. In the diagram, the dots are computed from the market prices of the out-of-the-money calls and puts. The solid line is generated by fitting the market implied volatility with a quadratic function that passes through the point at the money and minimizes the volume-weighted mean squared errors of the implied volatility. The bar chart is the trading volume normalized by 20,000 contracts for the corresponding options traded on November 4, 2003. 


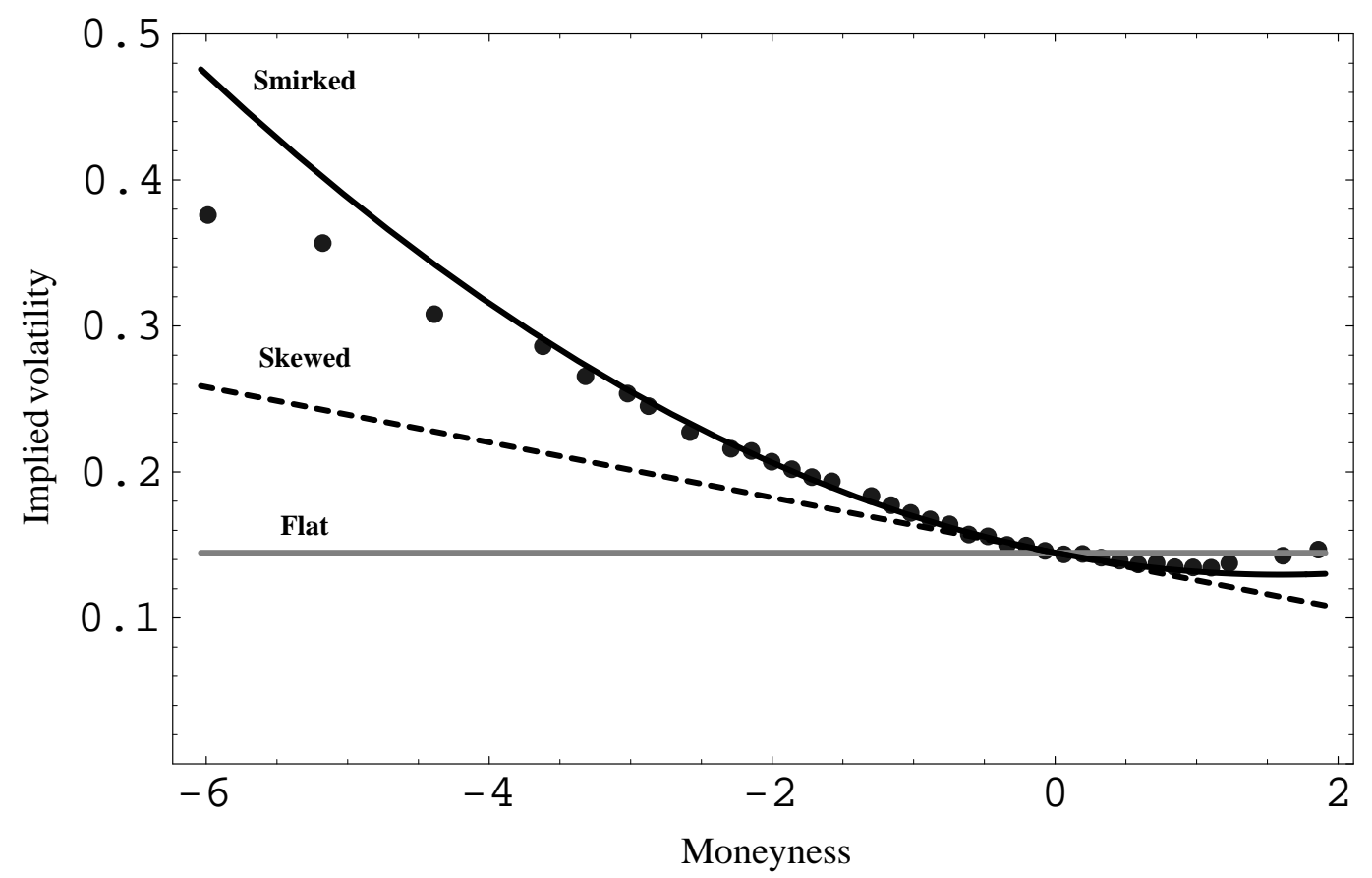

Figure 4: The flat, skewed and smirked implied volatility functions together with market implied volatilities (shown as dots) on November 4, 2003 for SPX options that mature on November 21, 2003. The flat volatility function is $I V=0.1447$, which is the ATMV. The skewed one is a linear volatility function, $I V=0.1447(1-0.1308 \xi)$. The smirked one is a quadratic function, $I V=0.1447\left(1-0.1308 \xi+0.0411 \xi^{2}\right)$. 

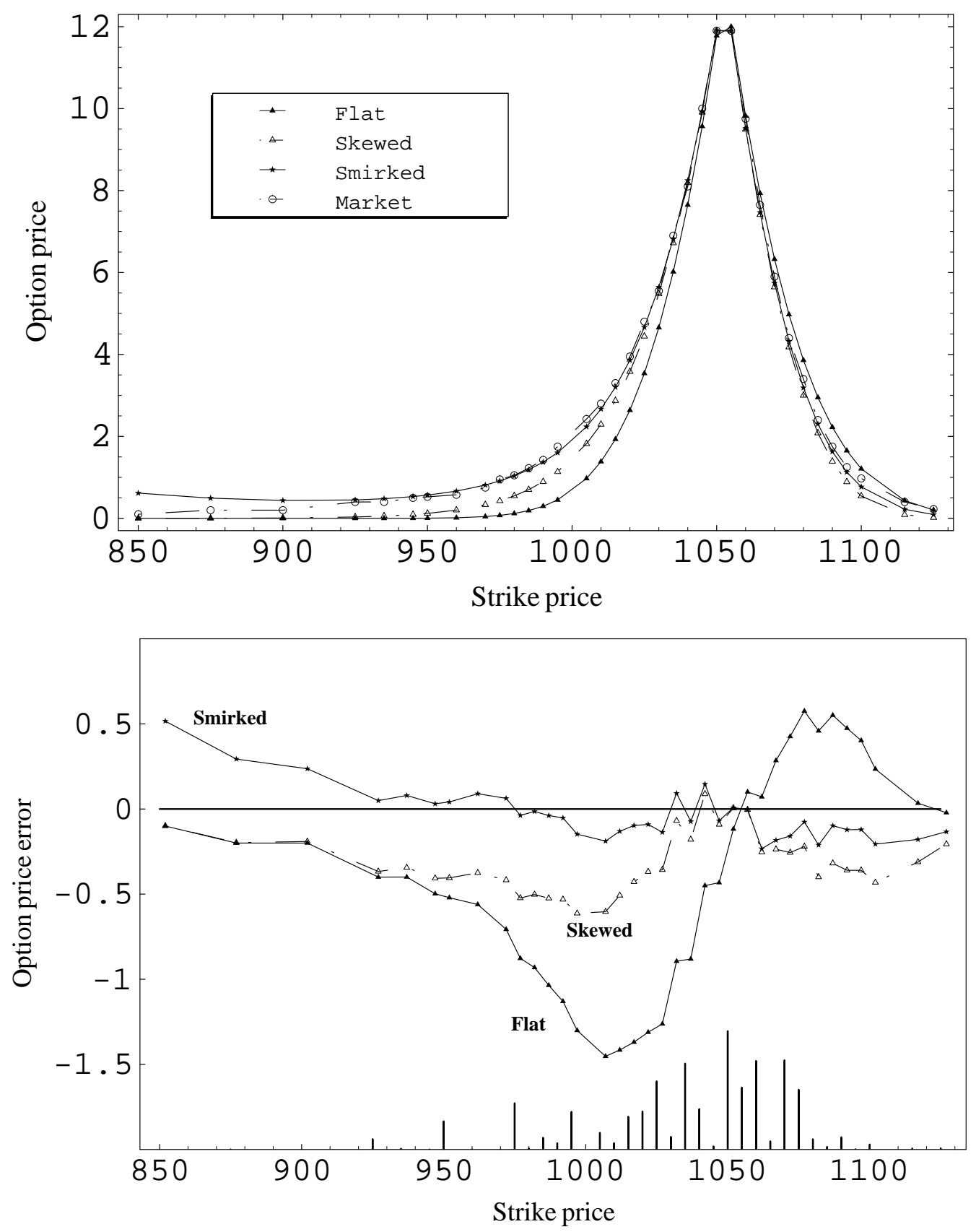

Figure 5: The computed option price and its error as functions of the strike price on November 4, 2003 for out-of-the-money SPX options that mature on November 21, 2003. The computed option price is determined by using the Black-Scholes formula with flat, skewed and smirked volatility functions. The flat one is $I V=0.1447$, which is the ATMV. The skewed one is a linear volatility function, $I V=0.1447(1-0.1308 \xi)$. And the smirked one is a quadratic function, $I V=0.1447\left(1-0.1308 \xi+0.0411 \xi^{2}\right)$. The price error is the difference between the computed price and the market price. At the money, $K=F_{0}=$ 1052.70, three computed option prices are the same as the market price. Errors are therefore zero. The option price error is shown together with the trading volume normalized by 5,000 contracts. 

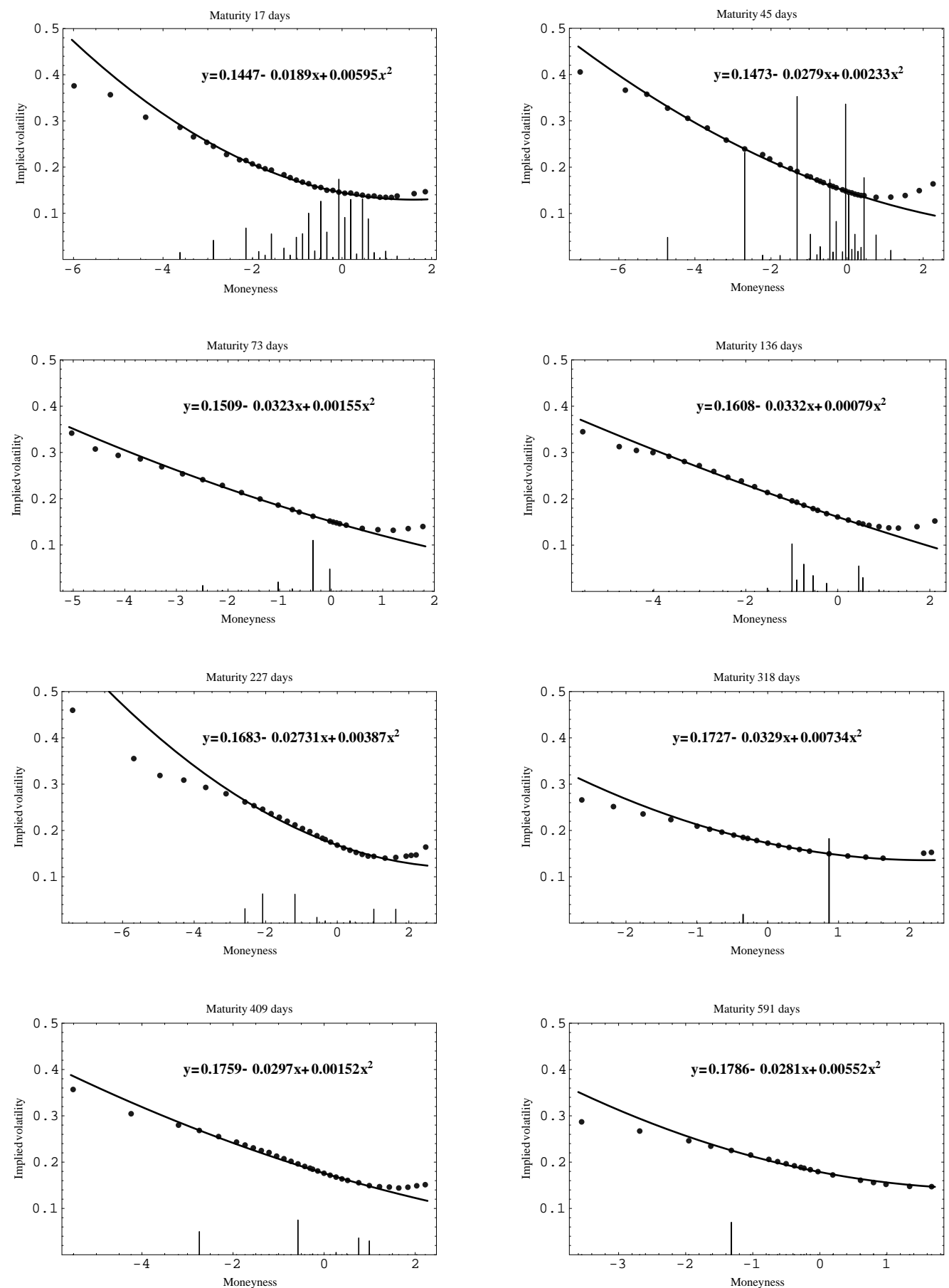

Figure 6: The implied volatility smirks on November 4, 2003 for options with all available maturities, including Nov-21-03, Dec-19-03, Jan-16-04, Mar-19-04, Jun-18-04, Sep-17-04, Dec-17-04, and Jun-17-05. The times to maturity are 17, 45, 73, 136, 227, 318, 409, and 591 days, respectively. The dots are computed from the market prices of the out-of-themoney calls and puts. The solid line is generated by fitting the market implied volatility with a quadratic function that passes through the point at the money and minimizes the volume-weighted mean squared errors of the implied volatility. The bar chart is the trading volume normalized by 20,000 contracts for the corresponding options traded on November 4, 2003. 

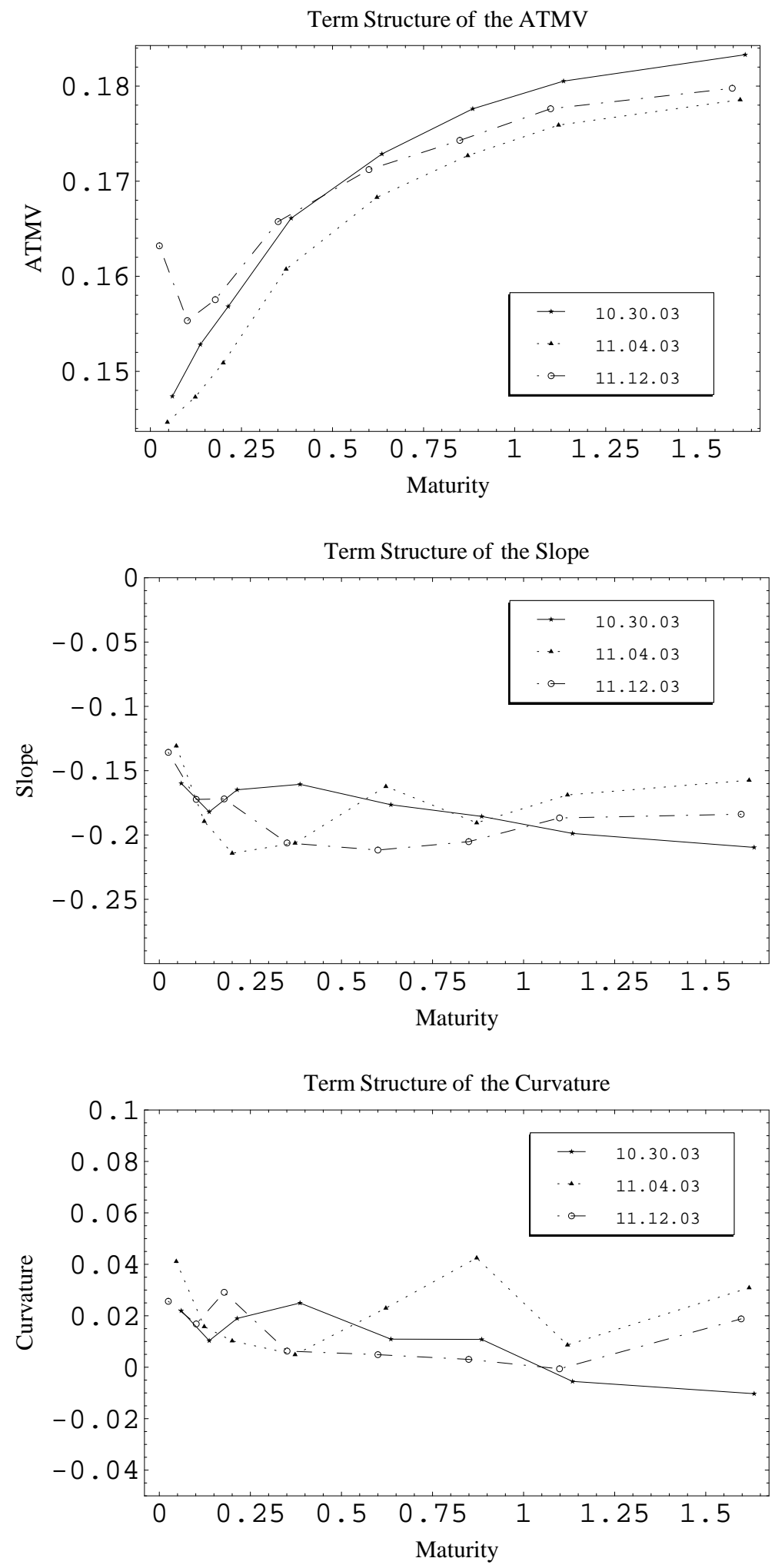

Figure 7: The evolution of the term structure of the implied volatility smirk parameters from October 30, 2003 to November 4, 2003 and then to November 12, 2003. The smirk parameter set $\left(\gamma_{0}, \gamma_{1}, \gamma_{2}\right)$ is obtained by fitting the implied volatility smirks with a quadratic function, $I V(\xi)=\gamma_{0}\left(1+\gamma_{1} \xi+\gamma_{2} \xi^{2}\right)$, where $\gamma_{0}$ is the ATMV, $\gamma_{1}$ is the slope, $\gamma_{2}$ is the curvature, and $\xi$ is the moneyness. 

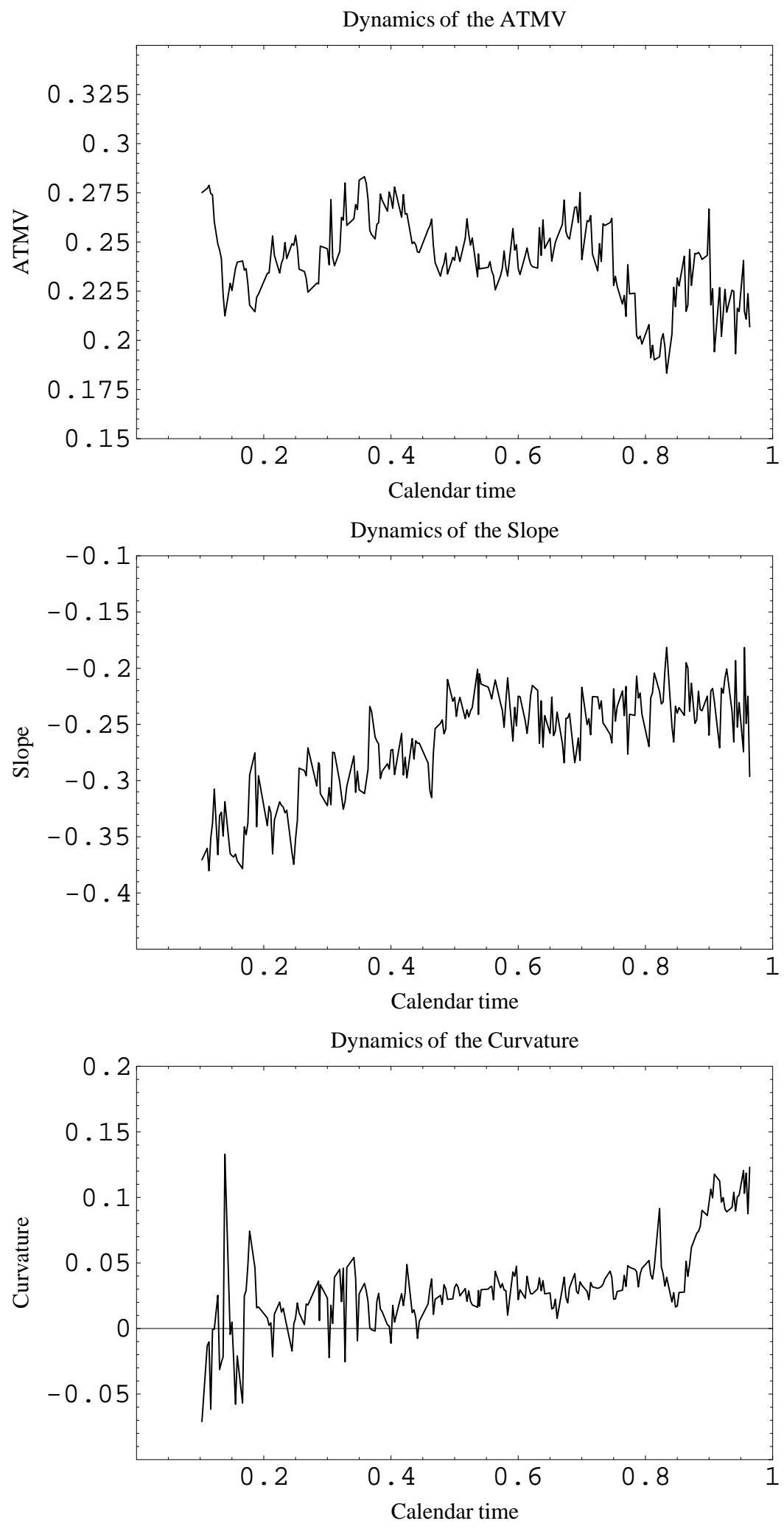

Figure 8: The time-change dynamics of the ATMV $\gamma_{0}$, the slope $\gamma_{1}$ and the curvature $\gamma_{2}$ of the implied volatility smirk for options with the maturity date, September 16, 1999. The time series is from September 25, 1998 to September 3, 1999. The horizontal axis is calendar time $t$ (year). The maturity date is at $t=1$. 

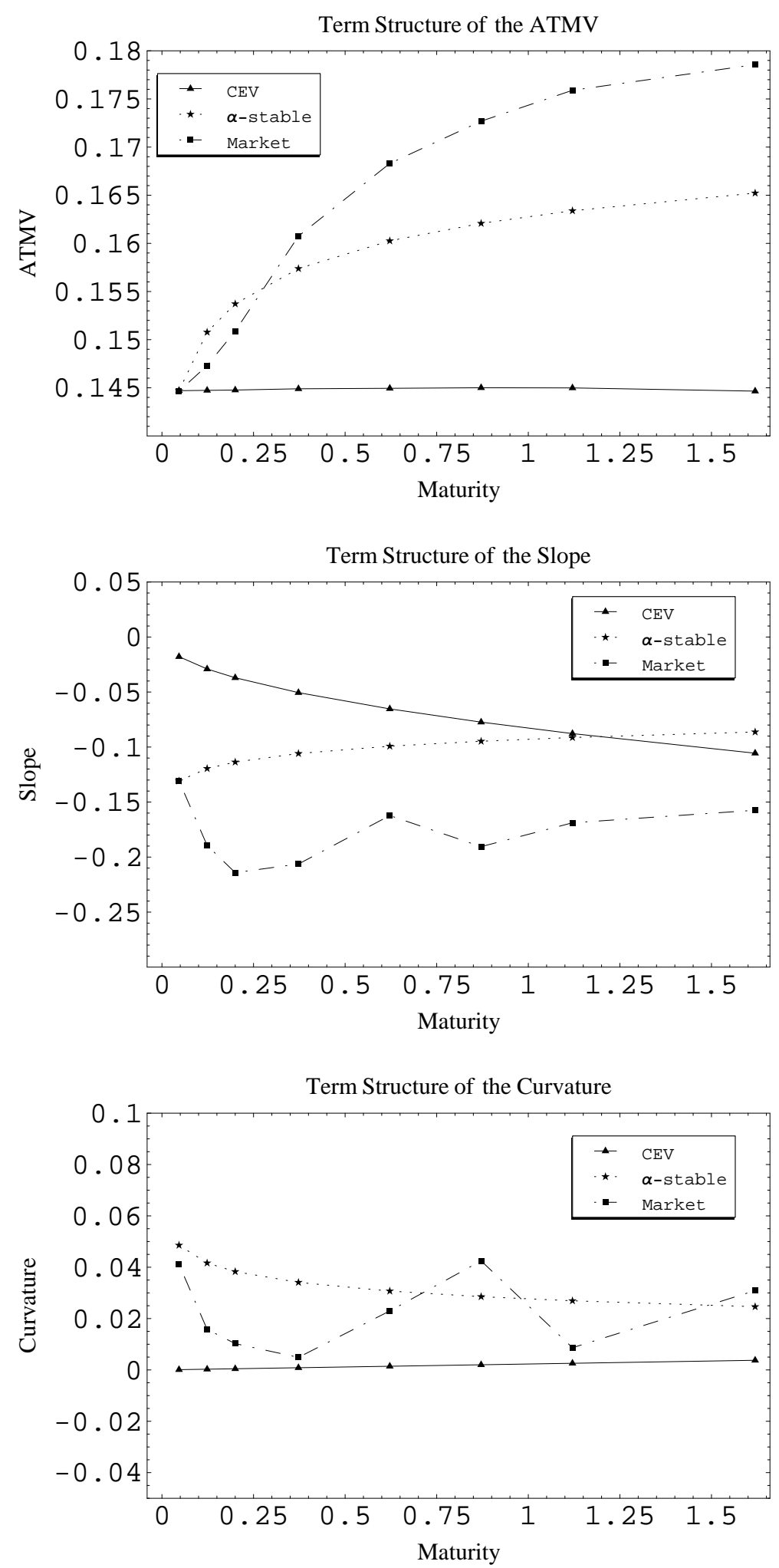

Figure 9: The term structure of smirk parameters implied in two calibrated option pricing models and observed from the market data on November 4, 2003. The two models are the Constant Elasticity of Variance (CEV) model, $d S_{t}=(r-q) S_{t} d t+\sigma S_{t}^{\alpha} d B_{t}$, with calibrated parameters: $(\sigma, \alpha)=(152.36,0)$, and the Finite Moment Log $\alpha$-Stable (FMLS) process, $d S_{t}=(r-q) S_{t} d t+\sigma S_{t} d L_{t}^{\alpha,-1}$, with calibrated parameters: $(\sigma, \alpha)=(0.1086,1.8141)$. 